Portland State University

PDXScholar

Winter 3-28-2019

\title{
Capillary Migration of Large Confined Drops in Non- wetting Wedges
}

Logan John Torres

Portland State University

Follow this and additional works at: https://pdxscholar.library.pdx.edu/open_access_etds

Part of the Fluid Dynamics Commons

Let us know how access to this document benefits you.

\section{Recommended Citation}

Torres, Logan John, "Capillary Migration of Large Confined Drops in Non-wetting Wedges" (2019). Dissertations and Theses. Paper 4825.

https://doi.org/10.15760/etd.6701

This Thesis is brought to you for free and open access. It has been accepted for inclusion in Dissertations and Theses by an authorized administrator of PDXScholar. Please contact us if we can make this document more accessible: pdxscholar@pdx.edu. 
Capillary Migration of Large Confined Drops in Non-wetting Wedges

\author{
by \\ Logan John Torres
}

A thesis submitted in partial fulfillment of the requirements for the degree of

\author{
Masters of Science \\ in \\ Mechanical Enginering
}

Thesis Committee: Mark Weislogel, Chair

Derek Tretheway

Gerald Recktenwald

Portland State University

2019 
(C) 2019 Logan John Torres 


\begin{abstract}
When confined within containers or conduits, drops and bubbles migrate to regions of minimum energy by the combined effects of surface tension, surface wetting, system geometry, and initial conditions. Such capillary phenomena are exploited for passive phase separation operations in micro-fluidic devices on earth and macro-fluidic devices aboard spacecraft. Our study focuses on the migration and ejection of large inertial-capillary drops confined between tilted planar hydrophobic substrates. In our experiments, the brief nearly weightless environment of a drop tower allows for the study of such capillary dominated behavior for up to $10 \mathrm{~mL}$ water drops with migration velocities up to $12 \mathrm{~cm} / \mathrm{s}$. We control ejection velocities as a function of drop volume, substrate tilt angle, initial confinement, and fluid properties. We then demonstrate how such geometries may be employed as passive nomoving-parts droplet generators for very large drop dynamics investigations. The method is ideal for hand-held non-oscillatory drop generation for fun, educational, and insightful astronaut demonstrations aboard the International Space Station.
\end{abstract}




\section{Dedication}

This work is dedicated to my Lord and Savior Jesus Christ, and to my family.

1 John 4:9 "In this the love of God was made manifest among us, that God sent his only Son into the world, so that we might live through him." 


\section{Acknowledgments}

This work was funded in part through NASA cooperative agreements NNX12AO47A and NNX16AC38G, and NIAC grant NNX15AR93G (Principle Investigator John Graf, NASA JSC).

I would like to thank Karl Cardin, Drew Wollman, Erin Schmidt, Rawand Rasheed, Isabel

Rodriguez, Caleb Turner, and Rihana Mungin for their advice, encouragement, and support throughout this project. Thanks to Quince Assenberg and Trent Anderson for their support in drop tower experiments.

And finally, I would like to thank Dr. Mark Weislogel. 


\section{Contents}

$\begin{array}{ll}\text { Abstract } & \text { i }\end{array}$

Dedication $\quad$ ii

Acknowledgments $\quad$ iii

List of Figures $\quad$ ix

1 Introduction 1

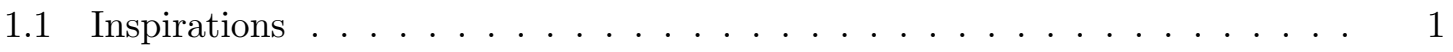

1.2 Puddle Jumping . . . . . . . . . . . . . . . . . . . . . . 2

1.3 Wedge ejector . . . . . . . . . . . . . . . . . . 4

2 Capillary migration in non-wetting wedges $\quad 8$

2.1 Methods . . . . . . . . . . . . . . . . . . . 8

2.2 Capillary driving force . . . . . . . . . . . . . . . . . . . . 10

2.3 Energy model . . . . . . . . . . . . . . . . . . . . . . . . 12

2.4 Initial conditions . . . . . . . . . . . . . . . . . . . 13

2.5 Results and Discussion . . . . . . . . . . . . . . . . . 16

2.6 Conclusions . . . . . . . . . . . . . . . . . . . . . . . . . . . 19

3 Applications and Future Work $\quad 22$

3.1 Future planar wedge drop migration investigations $\ldots \ldots \ldots$. . . . . . 22

3.2 Wedge drop generating devices . . . . . . . . . . . . . . . . . 24

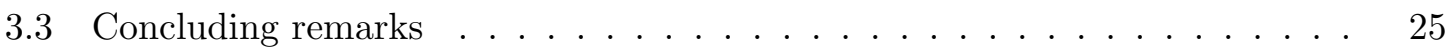


Appendix

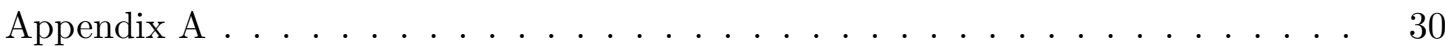

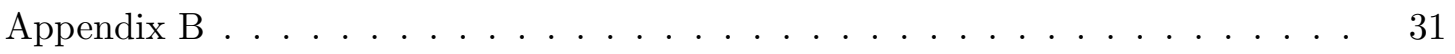




\section{List of Figures}

1 Time sequence $(20 \mathrm{~Hz})$ of a $5 \mathrm{~mL}$ dyed water puddle jump from a textured PTFE coated hydrophobic substrate with $\theta=150^{\circ}$ following the step reduction in gravity during a simple drop tower test. Sequence of images demonstrate the following: a. static 1-g interface, b. inward radial capillary wave formation, c. geyser formation and generation of satellite drop ejections, d.-f. large amplitude deflections, and g. drop detachment at steady velocity $U_{j} \ldots \ldots \ldots \ldots \ldots$

2 a. Snapshopt of $0.5 \mathrm{~mL}$ puddle jumps from curved hydrophobic surfaces. b. Drop jump velocity $U_{j}$ as a function of surface curvature $\mathcal{R}$ (courtesy of Attari et al. (2016)).

3 Time sequence $(20 \mathrm{~Hz})$ of repeated $2 \mathrm{~mL}$ blue dyed water puddle jumps in comparison to numerical analysis (red) conducted by Avhad et al. (2017) using open source software Gerris. The ejection velocity, time, oscillation frequency, and oscillation amplitude are found to be captured well. . . . . . . . . . . . . . .

4 Time sequence of a $5 \mathrm{~mL}$ puddle jump in hydrophobic wedge of $\alpha=2.5^{\circ}$ during a drop tower experiment. a. Static 1-g interface, b.-d. acceleration under internal capillary pressure driven flow, and e. ejection within $t=1.92 \mathrm{~s}$ with steady velocity $U_{w} \approx 10 \mathrm{~cm} / \mathrm{s} \ldots \ldots \ldots \ldots \ldots \ldots \ldots \ldots$

5 a. Experiment rig b. suspended within a drag shield, c. placed in the drop tower. The rig falls within the drag shield which falls $22.2 \mathrm{~m}$ in the drop tower providing $t_{\text {drop }}=2.1 \mathrm{~s}$ of near weightlessness with accelerations $\lesssim 10^{-4} g_{o} \ldots \ldots \ldots$

6 Wedge test cell with exploded view. Components include light diffuser, light shield, laser-cut wedge geometry mounting plates, and super-hydrophobic surfaces. The components assemble together to produce a test cell of predetermined $\alpha . \quad$. . . . 
$7 \quad$ Idealized capillary drop migration in a super-hydrophobic wedge with (1) initial, (2) confined transient, and (3) inscribed states. Characteristic curvatures $R_{1}$ and $R_{3}$ approximate the capillary pressure gradient inside the drop. Advancing and receding menisci represented by $x_{a}$ and $x_{r}$ are referenced from the wedge vertex. Initial and inscribed locations of the receding edge are identified by $x_{r_{o}}$ and $x_{r_{i n s}}$. The 'average' drop location is defined as $x_{a v e}=\left(x_{a}+x_{r}\right) / 2 \ldots \ldots \ldots \ldots$

8 a. Actual and b. simplified models in 1 confined and 2 inscribed states. The free inscribed drop of radius $R_{s}$ attains velocity $U_{w} \ldots \ldots \ldots \ldots$

9 Effect of wedge angle $\beta$ relative to gravity on oscillation amplitude and frequency at ejection location: a. numerically computed initial shapes of $1 \mathrm{~mL}$ drops in hydrophobic wedges of $\theta=150^{\circ}$ for $\alpha=4^{\circ}$ and $\beta=0^{\circ}, 45^{\circ}$, and $86^{\circ}$, b. drop tower experiments of cases in a. . . . . . . . . . . . . .

$1010 \mathrm{~Hz}$ images from drop tower tests of $2 \mathrm{~mL}$ drops between parallel hydrophobic surfaces with varying separation height $H_{w}$. The computed time constant $\tau=$ $3.91 / \zeta \omega_{n}$ for planar area oscillation decay decreases with the separation height $H_{w}$ as predicted by the viscous time scale $t_{\nu} \sim H_{w}^{2} / 4 \nu \ldots \ldots \ldots \ldots$

11 Transient location of averaged drop position $x_{\text {ave }}(\mathrm{cm})$ vs $t(\mathrm{~s})$ for wedges $1.0^{\circ} \leq$ $\alpha \leq 3.8^{\circ}$ and drops $0.5 \leq V \leq 10 \mathrm{~mL}$ with varying initial locations resulting in confinements $0.1 \leq x_{o}^{*} \leq 0.80$. Plots are arranged in order of increasing maximum drop velocity $U_{w}$ which generally increases with $x_{o}^{*}$. Inscribed drop locations are noted by red tick marks when achieved. Static equilibrium locations are noted by blue tick marks. . . . . . . . . . . . . . . . . . 
12 Normalized drop velocities $U_{w}^{*}=U_{w} / \tilde{U}$ versus capillary confinement parameter $x_{o}^{*}$. Open and solid symbols imply drop ejection or no-ejection, respectively. Small, closed dot-symbols without error bars identify theoretical velocities from the cylindrical puddle surface energy difference approximation of Eq. 2. An approximate linear trend in both experimental and theoretical velocities tend towards agreement

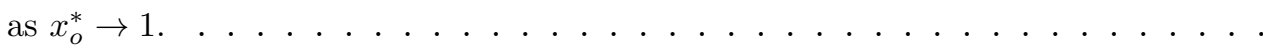

13 Experimental and theoretical ejection times $t_{w}$ with respect to the capillary confinement parameter $x_{o}^{*}$ with a. Eq. 3, b. Eq. 7, and c. Eq. 8. . . . . . . . . .

14 a. Time sequence of a $0.2 \mathrm{~mL}$ drop in hydrophobic wedge of $\alpha=2.8^{\circ}$ during a drop tower experiment. By $t=1.4 \mathrm{~s}$ the drop has been slowed and stopped by dissipative forces. b. Snapshot at $t=0.58 \mathrm{~s}$ of $1 \mathrm{~mL}$ viscous drops ranging in kinematic viscosity from 1 to $89 \mathrm{cSt}$. Increased viscosity decreases the viscous time scale $t_{\nu}=L_{s}^{2} / \nu$, thus initiating early transition to constant velocity as well as decreased ejection velocity.

15 a. A conical drop generator is designed and $3 \mathrm{D}$ printed in $\mathrm{b}$. as two halves such that the internal surface of each can be coated with a hydrophobic treatment. c. A single snap shot of a $0.71 \mathrm{~mL}$ drop ejecting from the $\alpha=2^{\circ}$ cone with velocity

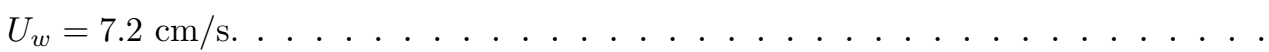

16 a. Example schematic of a continuous wedge drop generator through growth and detachment of drops at the apex of a planar hydrophobic wedge. b. Snapshot of drop tower experiment where a stream of $Q_{i n}=48 \mathrm{~mL} / \mathrm{min}$ of water is injected into an $\alpha=4^{\circ}$ wedge and rectified into drops of volume $V=0.29 \mathrm{~mL}$ with ejection velocity $U_{w}=10.2 \mathrm{~cm} / \mathrm{s} \ldots \ldots \ldots \ldots \ldots$ 
17 Simple schematic for a hand-held wedge drop generator. a. Fluid is injected between parallel hydrophobic surfaces until the desired volume is deposited. b. The upper surface is then mechanically rotated to the desired angle until in c. the deposited drop migrates and ejects under the internal capillary pressure from Eq. 1. . . . . 


\section{Nomenclature}

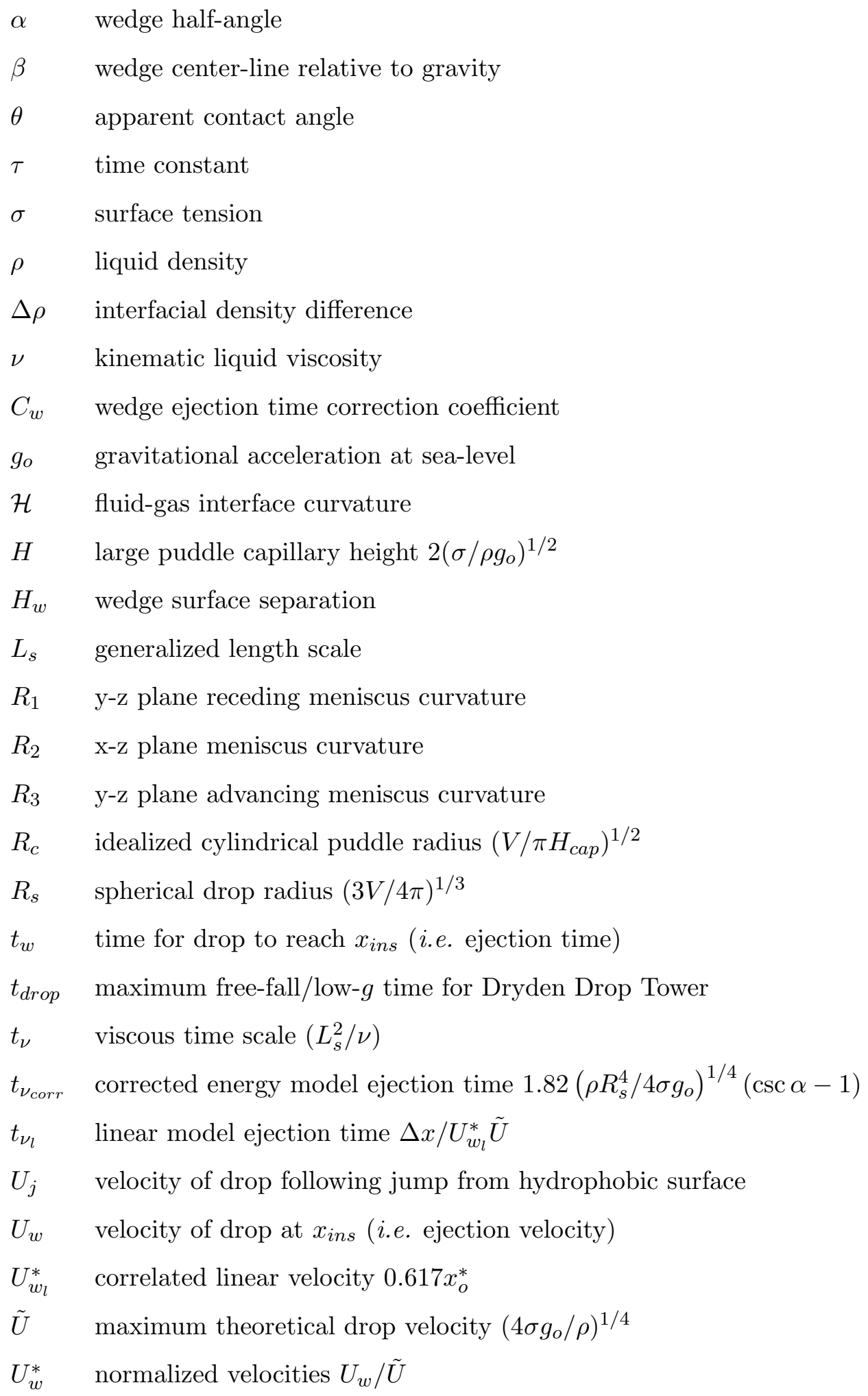




$\begin{array}{ll}V & \text { drop volume } \\ x_{r} & \text {-location of receding meniscus } \\ x_{a} & \text {-location of advancing meniscus } \\ x_{\text {ave }} & \text { averaged advancing and receding menisci }\left(x_{a}+x_{r}\right) / 2 \\ x_{c a p} & \text { receding edge minimum } x \text {-location in wedge } H / \cos \alpha \tan 2 \alpha \\ x_{r_{o}} & \text { initial } x \text {-location of receding meniscus } \\ x_{r_{i n s}} & \text { receding edge inscribed } x \text {-location } R_{s}(\csc \alpha-1) \\ \Delta x & \text { relative distance traveled } x_{r_{i n s}}-x_{r_{o}}\end{array}$




\section{Introduction}

\subsection{Inspirations}

Due to the absence of significant buoyancy in low-gravity environments, multi-phase fluid dynamics such as droplet laden flows are routinely found in spacecraft systems. Methods to limit or exploit the presence of such capillary dominated drops is relatively unresolved, and represents a large volume of low-gravity fluid research. Due to the overwhelming magnitude of gravitational forces compared to surface tensions forces on earth, capillarity is relegated to small scale systems of order $O(<1 \mathrm{~mm})$ as determined by the capillary

length scale $(\sigma / \Delta \rho g)^{1 / 2} \equiv l_{\text {cap }}$ where $\sigma$ is the fluid surface tensions, $\Delta \rho$ is the fluid interface density difference, and $g$ is gravity. Consequently, capillary phenomena at these scales occur over short time periods and generally in viscous dominated regimes. In contrast, reduced gravity environments extend the capillary length scale as much as 1000-fold, coincidentally increasing the time for capillary dynamics to develop. Thus, to experimentally observe and investigate low- $g$ capillary drop dynamics, experiments must be conducted on-board orbiting space labs such as the International Space Station (ISS), in parabolic flights, or in drop towers (Langbein (2002)) where gravity is commonly reduced to $\lesssim O\left(10^{-6} g\right)$.

However, even within these environments, the generation of large liquid drops for experiments can be a non-trivial task. For example, a common method for drop deployment involves the inflation and detachment of a pinned drop at the tip of a syringe needle (Robinson and Chai (1997)). During inflation, the drop may become de-pinned from the needle tip and wet the outside surface causing failure of the deployment system. Additionally, during detachment the rupturing of the liquid bridge between the pinning sight and the 
drop induces surface disturbances which may be undesirable for the ensuing experiment.

Other methods such as jet breakup and acoustic levitation experience similar and more amplified surface disturbances. A single method to generate large quiescent slow moving drops on demand is still a desired capability. The following research demonstrates a novel method, referred to here as the wedge drop generator, to address this challenge. Using the short-duration low-gravity environment of the Dryden Drop Tower experimental work was conducted in an effort to demonstrate such wedge drop generators. The following sections review preliminary and supporting research related to puddle jumping including its relationship to the wedge drop generator, a fundamental review of the driving forces for the drop migration phenomena, a preliminary analysis of drop ejection velocities and ejection times using first principle concepts, experimental investigation of wedge drop generators, and finally future work and applications of the wedge drop generator.

\subsection{Puddle Jumping}

Recent studies by Wollman et al. (2016) and Attari et al. (2016) demonstrate a method for large droplet ejection from puddles that jump spontaneously from hydrophobic surfaces during routine drop tower tests. Figure 1 illustrates such jumping for a $5 \mathrm{~mL}$ water puddle on a textured PTFE surface with static contact angle of $\theta=150^{\circ}$. Drop volumes provided from such 'puddle jumps' can be over 1000 times larger than similar terrestrial based drop bounce behavior (e.g., Richard and Quéré (2000)). The sudden reduction in gravity initiates a radially inward capillary wave that constructively interferes at the drop axis resulting in a variety of events which may include geyser formation, satellite drop ejection, and bubble ingestion followed by significant vertical elongation of the bulk fluid and subsequent 


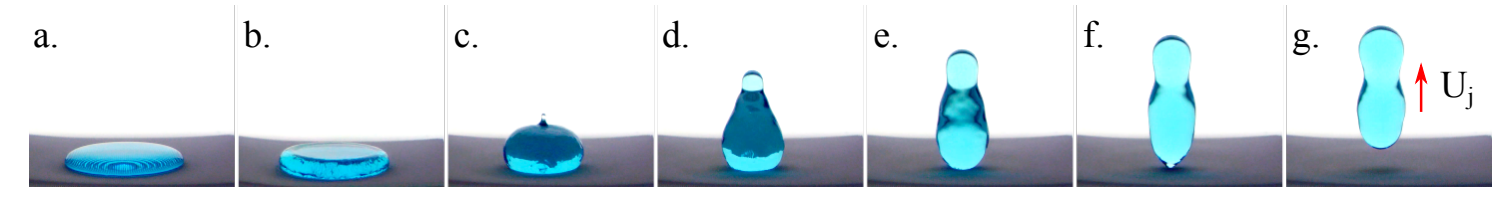

Figure 1: Time sequence $(20 \mathrm{~Hz})$ of a $5 \mathrm{~mL}$ dyed water puddle jump from a textured PTFE coated hydrophobic substrate with $\theta=150^{\circ}$ following the step reduction in gravity during a simple drop tower test. Sequence of images demonstrate the following: a. static 1-g interface, b. inward radial capillary wave formation, c. geyser formation and generation of satellite drop ejections, d.-f. large amplitude deflections, and g. drop detachment at steady velocity $U_{j}$.

detachment from the hydrophobic surface (also known as 'jumping'). Many interesting phenomena related to large drop dynamics can be studied during the process. The behavior of non-wetting moving contact lines, highly inertial capillary surface oscillations, analogies to droplet rebound phenomena, and numerical method benchmarking are a few such studies. Additionally, such uncomplicated passive generation of large free droplets enables other unique studies in reduced gravity such as capillary effects near Leidenfrost points (Attari et al. (2016), Al Jubaree et al. (2016)), and electrostatic droplet manipulation studies (Schmidt and Weislogel (2016)). The puddle jumping method is particularly useful in drop tower tests due to its simple implementation.

The puddle jump experiments in Figures 2 and 3 represent work conducted by the author in support of the published data by Attari et al. (2016) as well as work presented and under preparation for publication by Avhad et al. (2017). In Figure 2a, ejection velocities for a drop of spherical radius $R_{D}$ is controlled by surface radius of curvature $R_{s}$. Figure $2 \mathrm{~b}$ presents jump velocity $U_{j}$ as a function of radius of curvature ratio $1-R_{d} / R_{s} \equiv \mathcal{R}$ through the linear approximation $U_{j}=0.103 \mathcal{R}+0.012$, where $U_{j}$ is in $\mathrm{m} / \mathrm{s}$. In Figure 3 , repeated 

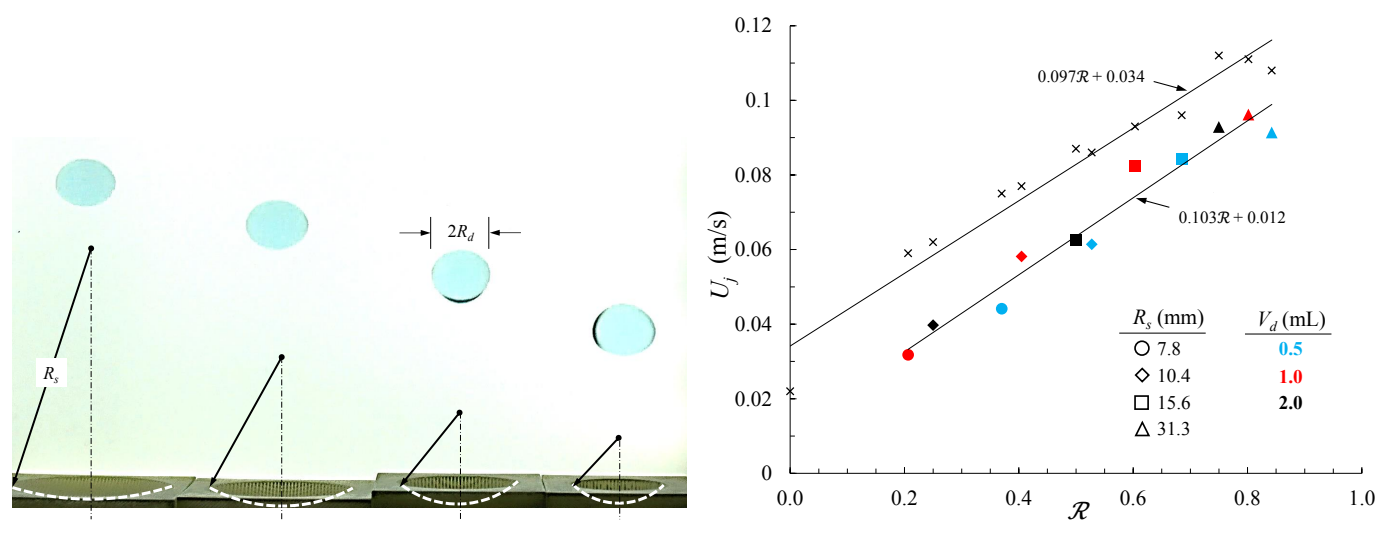

Figure 2: a. Snapshopt of $0.5 \mathrm{~mL}$ puddle jumps from curved hydrophobic surfaces. b. Drop jump velocity $U_{j}$ as a function of surface curvature $\mathcal{R}$ (courtesy of Attari et al. (2016)).

$2 \mathrm{~mL}$ puddle jumps serve as benchmarks for comparison to numerical analysis using open source software Gerris (Popinet (2003)) in Avhad et al. (2017). Characteristics such as jump velocity, jump time, and mode of oscillation are captured well in many cases.

\subsection{Wedge ejector}

Unfortunately, the large amplitude under-damped oscillations created during the puddle jump sequence add complexity to further interactions downstream. Methods to reduce such oscillations are limited without significant changes to fluid properties such as increased dynamic viscosity (Al Jubaree et al. (2016)). As demonstrated in Fig. 4, adapting the drop tower induced puddle jump mechanism, an alternate method of drop generation and ejection exploits the geometry of a hydrophobic wedge to provide a capillary pressure gradient to drive the partially confined liquid out of the wedge with drastically reduced oscillations. The wedge geometry is alternately referred to as an interior corner, interior edge, tapered channel, non-parallel plates, tilted plates, etc. in the literature. The passive migration of gas bubbles and liquid slugs in acute wedges has been studied extensively. Terrestrial research 


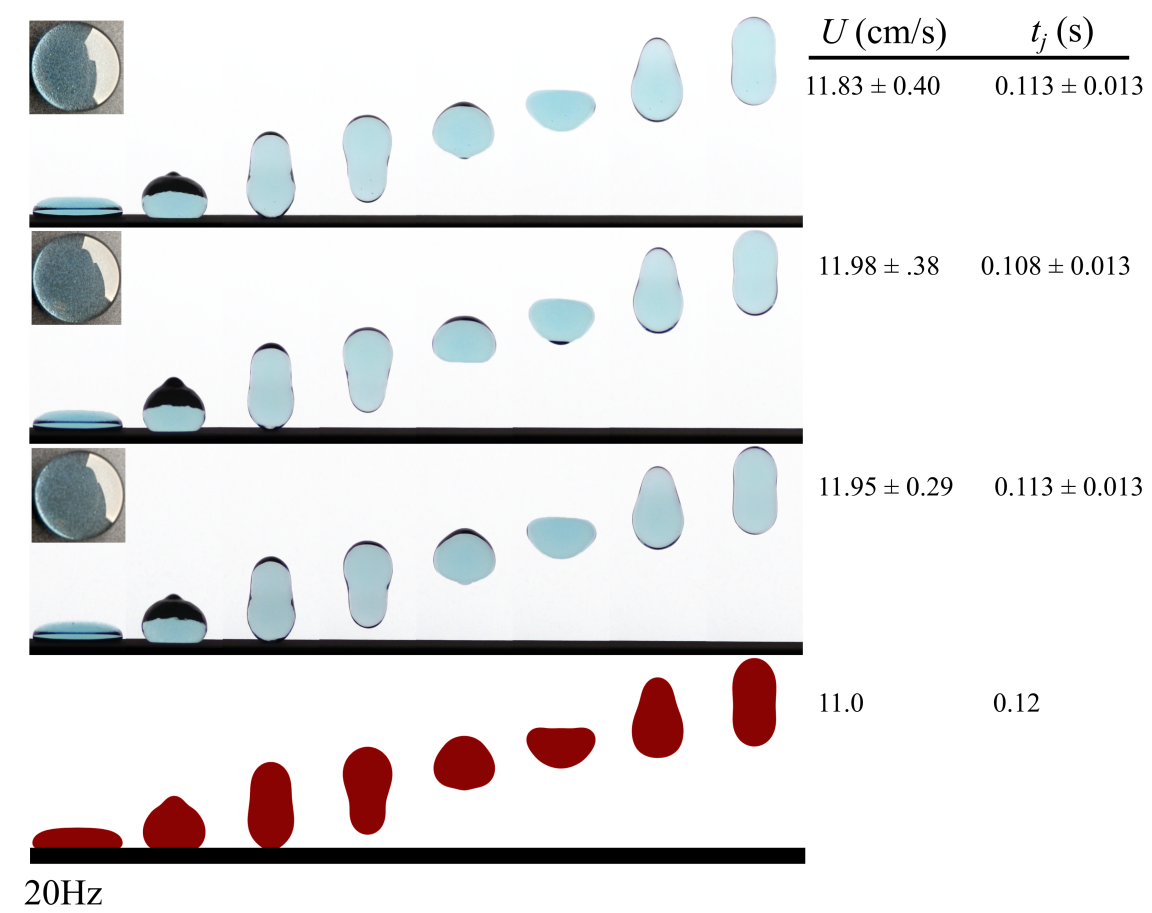

Figure 3: Time sequence $(20 \mathrm{~Hz})$ of repeated $2 \mathrm{~mL}$ blue dyed water puddle jumps in comparison to numerical analysis (red) conducted by Avhad et al. (2017) using open source software Gerris. The ejection velocity, time, oscillation frequency, and oscillation amplitude are found to be captured well.

regarding micro-fluidic applications is provided by Khare et al. (2007), Dangla et al. (2013), Luo et al. (2014), Luo and Heng (2014), Reyssat (2014), Baratian et al. (2015), Xu et al. (2016), and Ruiz-Gutiérrez et al. (2017) with demonstrations of passive bubble removal from multi-phase flows in micro-gravity provided by Jenson et al. (2014), Weislogel et al. (2009), and Weislogel et al. (2015). Due to the small length scales $L_{s}$ associated with terrestrial capillarity $L_{s} \lesssim(\sigma / \Delta \rho g)^{1 / 2}$ where $\Delta \rho$ is the density difference across the interface, $g$ is the magnitude of gravity, $L_{s}$ is the characteristic interface length scale, and $\sigma$ is the surface tension, viscous resistance and contact line boundary conditions often dominate the fluid 


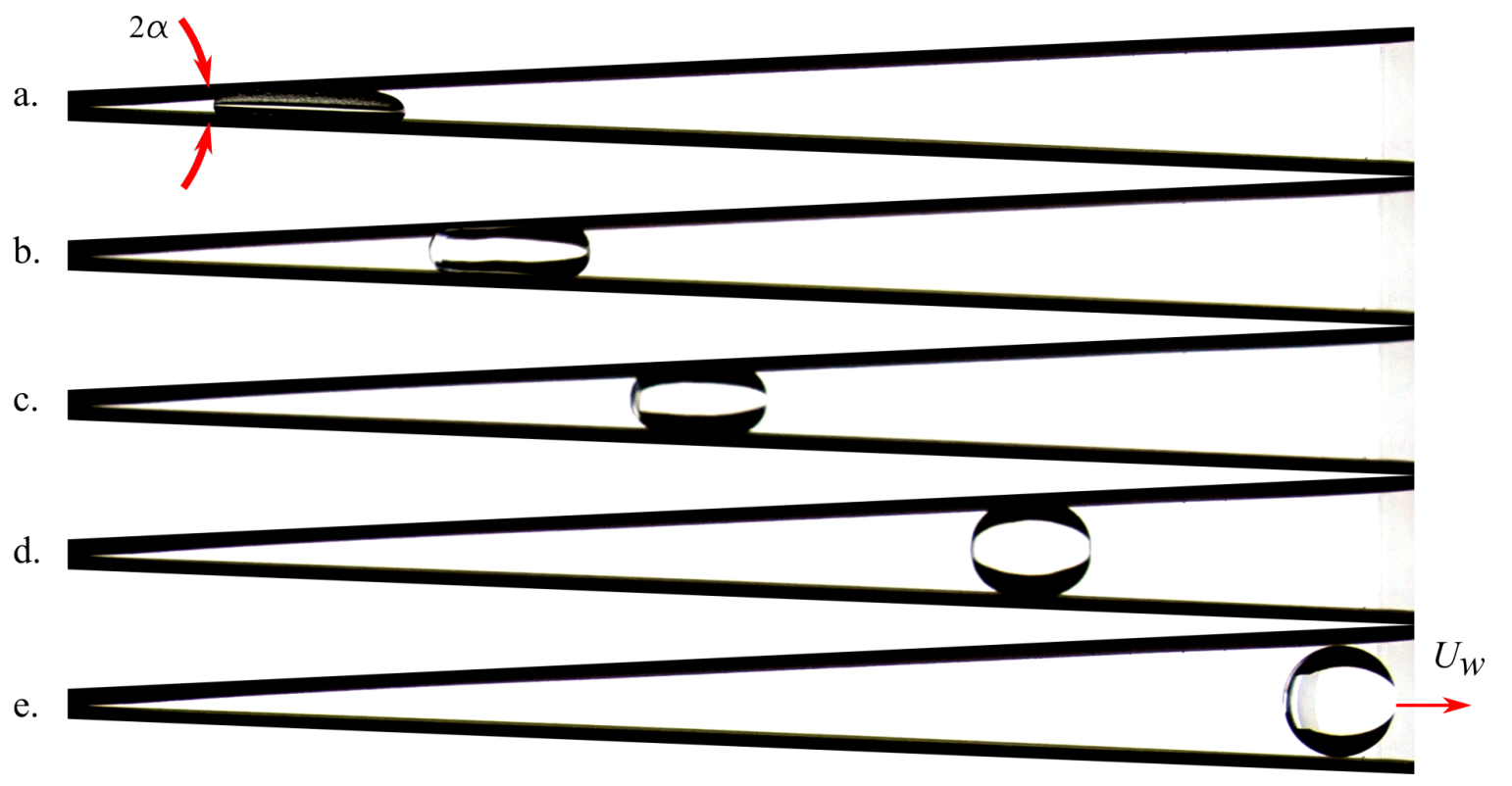

Figure 4: Time sequence of a $5 \mathrm{~mL}$ puddle jump in hydrophobic wedge of $\alpha=2.5^{\circ}$ during a drop tower experiment. a. Static 1-g interface, b.-d. acceleration under internal capillary pressure driven flow, and e. ejection within $t=1.92 \mathrm{~s}$ with steady velocity $U_{w} \approx 10 \mathrm{~cm} / \mathrm{s}$.

behavior. By contrast in micro-gravity environments, body forces are significantly reduced allowing capillary forces to extend their influence 1000-fold. As a result, potentially useful devices for passive fluid operations such as the wedge geometry can be applied over large length scales $\sim O(1 \mathrm{~m})$.

Due in part to the limited access to low-g environments, less attention has focused on inertial-capillary dominated liquid drops in non-wetting wedges satisfying $\theta>\pi / 2+\alpha$ (Concus and Finn (1998)) where $\theta$ is the equilibrium contact angle of the surface and $\alpha$ the wedge half-angle. Drop towers provide short free-fall periods for low-g experimentation to study such inertial-capillary wedge migration. The short term goal of this study is to observe how the wedge geometry controls ejection velocity for such large drops, while considering the limited time for ejection afforded by drop towers. The results include design guidelines 
for large quiescent drop generators for follow-on research conducted in drop towers as well as in other reduced gravity facilities (i.e., parabolic aircraft and spacecraft). 


\section{Capillary migration in non-wetting wedges}

\section{$2.1 \quad$ Methods}

All experiments are conducted using the $2.1 \mathrm{~s}$ Dryden Drop Tower located at Portland State University depicted schematically in Fig. 5. The $22.2 \mathrm{~m}$ tall tower provides a brief $t_{d r o p} \approx 2.1 \mathrm{~s}$ free fall with maximum acceleration $\lesssim O\left(10^{-4} g_{o}\right)$. The test rig shown in Fig. 5a contains all necessary experiment components including a wedge test cell, diffuse LED backlight panel, batteries, and a HD Panasonic HC-WX970 video camera. The test rig experiences low air resistance during its decent due to the use of a drag shield.

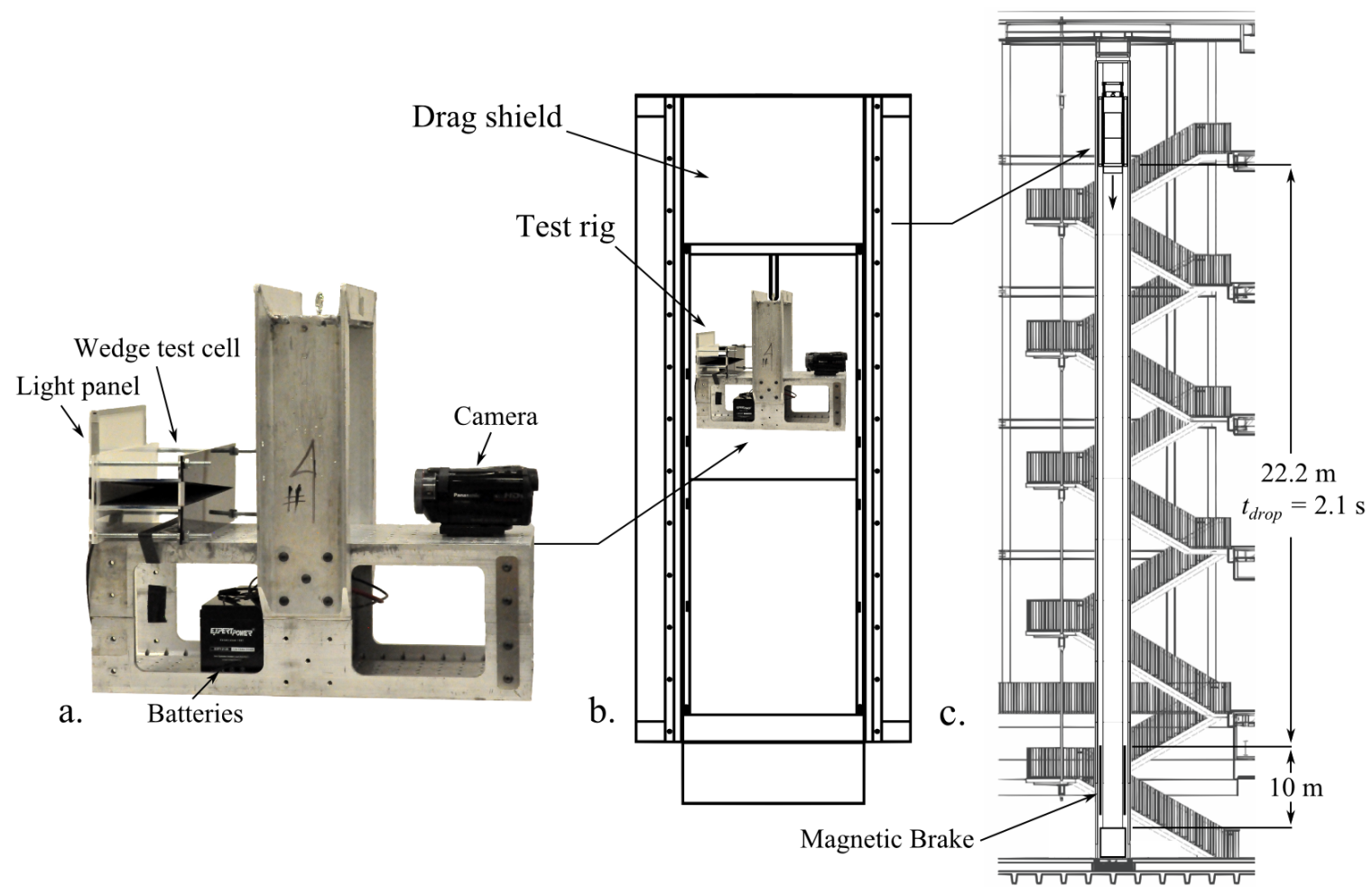

Figure 5: a. Experiment rig b. suspended within a drag shield, c. placed in the drop tower. The rig falls within the drag shield which falls $22.2 \mathrm{~m}$ in the drop tower providing $t_{\text {drop }}=2.1 \mathrm{~s}$ of near weightlessness with accelerations $\lesssim 10^{-4} g_{o}$. 
A typical wedge test cell is shown in exploded view in Fig. 6. Aluminum Oxide 320-grit sandpaper adhered to a metal plate via transfer tape is spray-coated with a polytetrafluoroethylene-based (PTFE) commercial aerosol spray (King Controls Magic Dome) to create planar hydrophobic substrates with average apparent static contact angles of $\theta=147 \pm 3^{\circ}$, as determined by height-width measurements of 10 low Bond number sessile drops over each surface. The assembled wedge test cell is fixed to the test rig with the lower wedge side perpendicular to gravity.

With the assembled test rig and test cell hanging inside the drag shield, a drop of distilled water is deposited onto the horizontal wedge side using a graduated syringe and rolled into the desired wedge location by slightly pitching and rolling the test rig and cell.

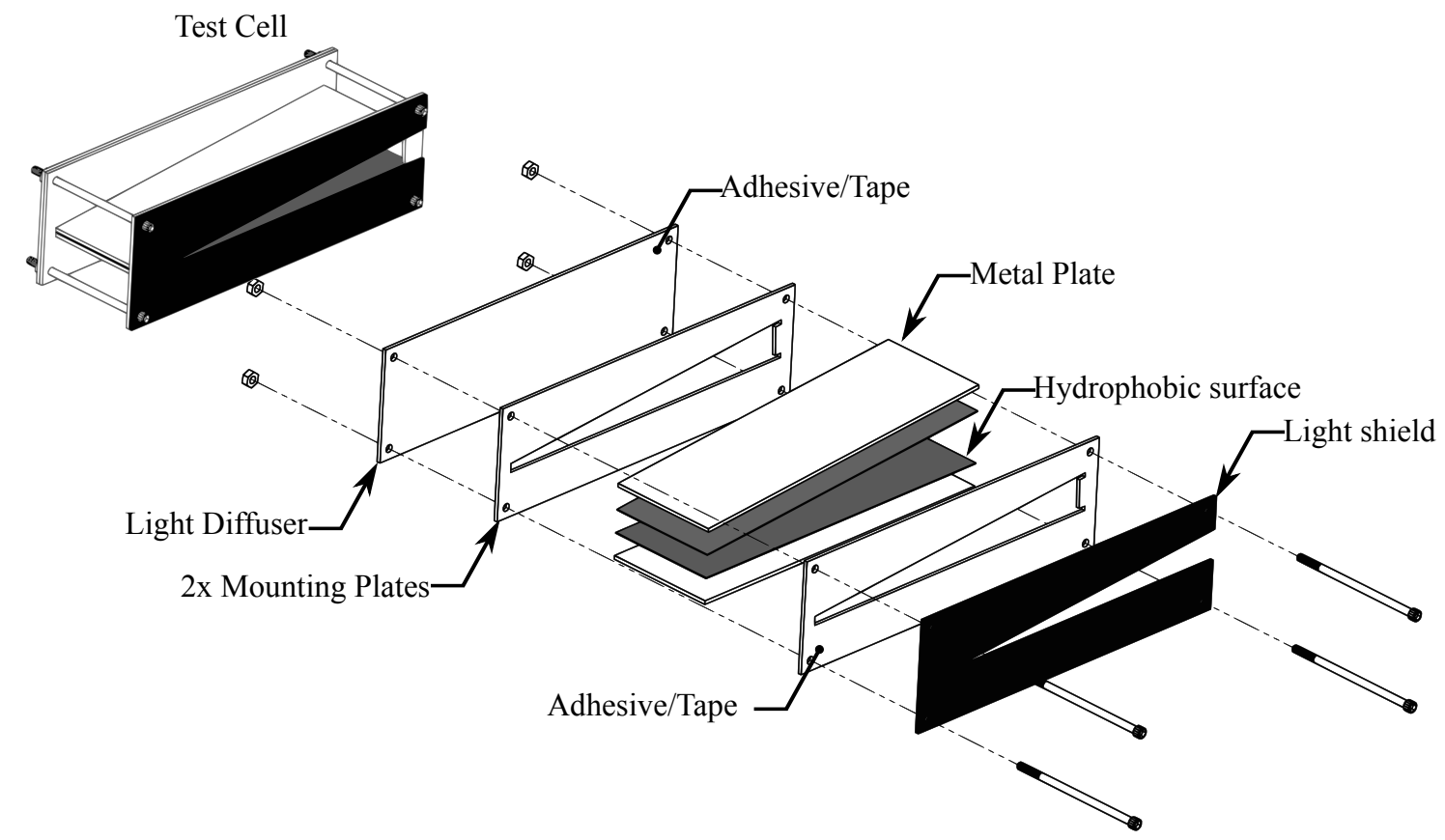

Figure 6: Wedge test cell with exploded view. Components include light diffuser, light shield, lasercut wedge geometry mounting plates, and super-hydrophobic surfaces. The components assemble together to produce a test cell of predetermined $\alpha$. 
Up to $120 \mathrm{~Hz}$ images of the ensuing low-g phenomena are converted from continuous video captured during free-fall and later analyzed using the open-source image analysis software FIJI by Schindelin et al. (2012). Reduced data such as planar area and front, back, and centroid locations are gathered. Over 200 drops were conducted in support of this work. A selection of 29 of these tests are included in Appendix A (Tables A.2 and 3). These experiments will be discussed in detail in this report following a brief review of the driving forces of the phenomena.

\subsection{Capillary driving force}

The Young-Laplace equation $\Delta P=\sigma \mathcal{H}$ defines the pressure jump across a gas-liquid interface exhibiting surface tension $\sigma$ and local surface curvature $\mathcal{H}$. Applied to the idealized capillary puddle in Fig. 7, a net liquid phase pressure gradient can be written in terms of the principle radii of curvature

$$
\Delta P_{l} \approx \sigma\left(\frac{1}{R_{3}}-\frac{1}{R_{1}}\right)
$$

where $R_{1}$ and $R_{3}$ are the receding and advancing menisci radii of curvature, respectively. The radius $R_{2}$ is shared by both advancing and receding curvatures and cancels from Eq. 1. The approximation of Eq. 1 applies along the positive $x$-axis for uniform planar wedges and symmetric fluid bodies in the $x-y$ plane. It is this pressure gradient that leads to bulk motion away from the wedge vertex. Under low-g conditions, the gradient diminishes with $R_{1} \rightarrow R_{3}$ as the drop acquires an increasingly spherical configuration.

Concus and Finn (1998) proved the existence of a stable equilibrium point for static drops exhibiting static contact angle $\theta>\pi / 2+\alpha$ where the equilibrium radius $R_{1}=R_{3} \equiv$ $R_{e q}$ is a function of wedge geometry and wetting conditions $R_{e q}=f(\alpha, \theta, V)$ (refer to Fig. 7, 
a.

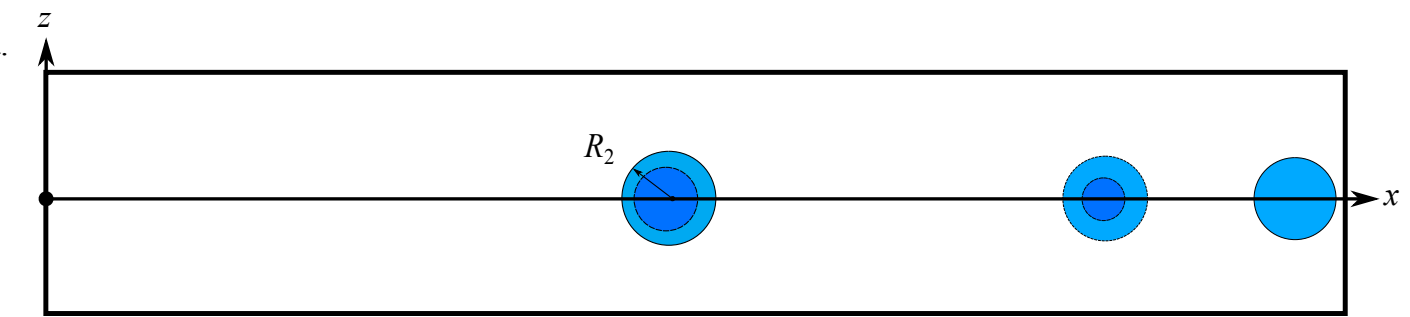

b.

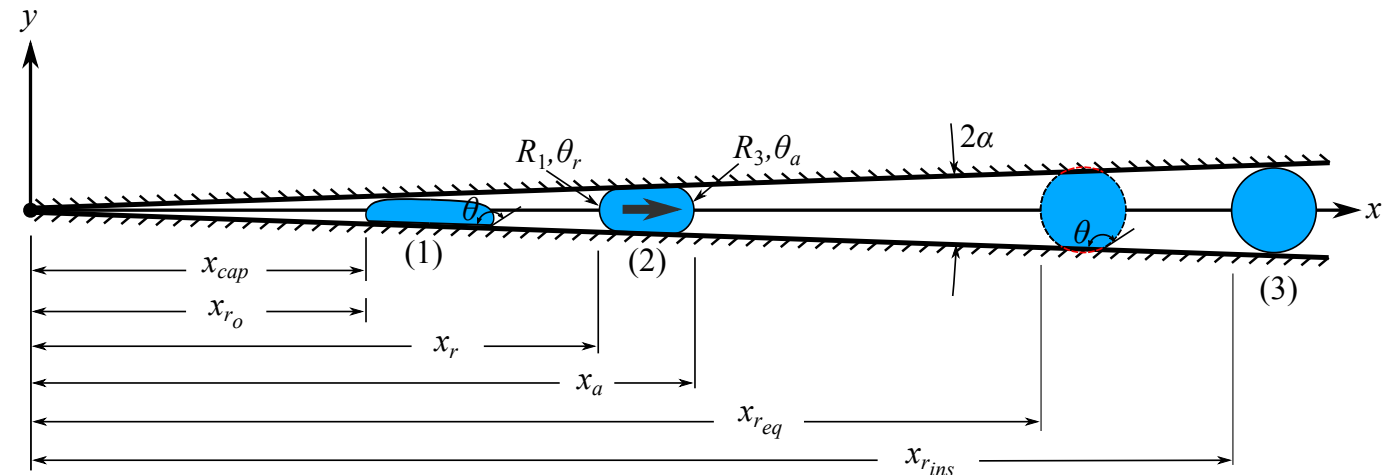

Figure 7: Idealized capillary drop migration in a super-hydrophobic wedge with (1) initial, (2) confined transient, and (3) inscribed states. Characteristic curvatures $R_{1}$ and $R_{3}$ approximate the capillary pressure gradient inside the drop. Advancing and receding menisci represented by $x_{a}$ and $x_{r}$ are referenced from the wedge vertex. Initial and inscribed locations of the receding edge are identified by $x_{r_{o}}$ and $x_{r_{i n s}}$. The 'average' drop location is defined as $x_{a v e}=\left(x_{a}+x_{r}\right) / 2$.

see also Langbein (2002), Baratian et al. (2015) and Ruiz-Gutiérrez et al. (2017)). However, for sufficiently large $\theta$, despite viscous resistance and finite dynamic contact angle hysteresis, inertial-capillary migrating drops routinely overshoot the confined equilibrium location $x_{r_{e q}}$ and achieve spherical states as they pass through the inscribed location $x_{r_{i n s}}=R_{s}(\csc \alpha-1)$ where the drop is tangent to both wedge surfaces. Thus, for the dynamic drops of this study, Eq. 1 applies when $x_{r}<x_{r_{\text {ins }}}$. 


\section{$2.3 \quad$ Energy model}

In a similar manner to Attari et al. (2016) and numerous others, a simplified energy model requires minimal analytical effort to gather insight relating to drop ejection transients and ejection velocity limits. Refering to Fig. 8a, the surface energy $(S E)$ difference from confined state 1 to free state 2 is converted to kinetic energy $(K E)$ of the bulk fluid via $K E_{2}=S E_{1}-S E_{2}$, ignoring work and dissipation during the transition. In the limit of small wedge half-angle $\alpha$ and large volumes $V$ the non-axisymetric interface configuration of Fig. 8a 1 is approximated by the axisymetric cylindrical disc configuration of Fig. 8b 1. Deriving the surface energies for the simplified states and solving for the bulk velocity (see Appendix B), a modified version of the jump velocity from Attari et al. (2016) is found to be

$$
U_{w}=\tilde{U}\left[-\cos \theta+\left(\frac{\pi H^{3}}{V}\right)^{1 / 2}-\frac{6^{2 / 3}}{2}\left(\frac{\pi H^{3}}{V}\right)^{1 / 3}\right]^{1 / 2}
$$

where $H \equiv 2\left(\sigma / \rho g_{o}\right)^{1 / 2}$ is the capillary height and $\tilde{U} \equiv\left(4 \sigma g_{o} / \rho\right)^{1 / 4}$ is the maximum theoretical velocity of the drop in the large puddle limit $\left(\pi H_{c a p}^{3} / V\right)^{1 / 3} \ll 1$ for $\theta \approx 180^{\circ}$. Dividing the maximum distance the fluid must travel to reach its inscribed length $x_{r_{\text {ins }}}$ by the maximum ejection velocity $\tilde{U}$ provides a characteristic ejection time

$$
t_{w}=\left(\frac{\rho R_{s}^{4}}{4 \sigma g_{o}}\right)^{1 / 4}(\csc \alpha-1),
$$

where again $R_{s}=(3 V / 4 \pi)^{1 / 3}$. The simplified forms of Eqs. 2 and 3 serve as design guides for wedge drop generators where a specified ejection velocity is desired and where the duration of free fall is limited. The experimental validation of such predictions and their underlying assumptions are investigated in part here. 
a.

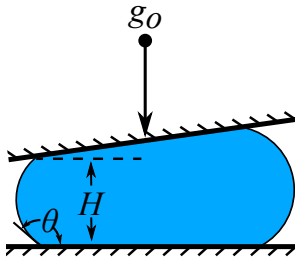

1

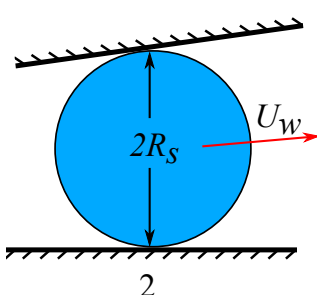

2

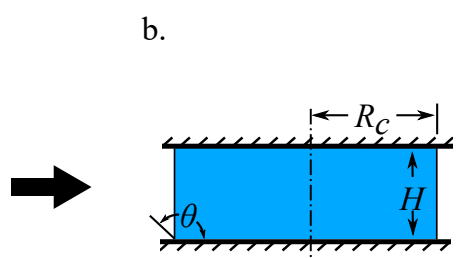

1

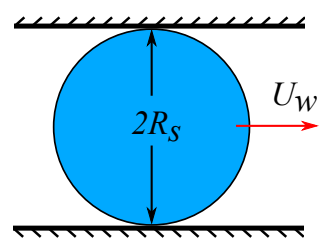

2

Figure 8: a. Actual and b. simplified models in 1 confined and 2 inscribed states. The free inscribed drop of radius $R_{s}$ attains velocity $U_{w}$.

\subsection{Initial conditions}

In the drop tower experiments, a puddle of known volume is deposited at some initial position $x_{r_{o}}<x_{r_{i n s}}$ within a hydrophobic wedge. A fixed drop position is assured when Eq. 1 is either zero or balanced by another force (i.e., tilt with respect to gravity). The initial conditions are met by varying the wedge center-line angle relative to gravity as shown in Fig. 9a, the former case occurring at $\beta=90^{\circ}-\alpha \equiv \beta_{\perp}$ and the latter over $0^{\circ} \equiv \beta_{\|} \leq \beta<\beta_{\perp}$. When $\beta=\beta_{\perp}$, gravity is perpendicular to the lower face of the wedge allowing the large drop to establish a symmetric disk-like puddle of capillary height $H \approx 2\left(\sigma / \rho g_{o}\right)^{1 / 2}$ when $V \gg\left(\sigma / \Delta \rho g_{o}\right)^{3 / 2}$. For $\beta_{\|} \leq \beta<\beta_{\perp}$, the drop is drawn into the vertex to various degrees by its own weight resisted only by the capillary pressure gradient Eq. 1. The drop elongates along the wedge vertex reaching a maximum length when $\beta=\beta_{\|}$.

Following the effective step-reduction in gravity of the drop tower tests, reorientation of the $\beta=\beta_{\perp}$ initial condition follows that of puddle jumping (ref. Fig. 1). However, the upper surface of the wedge suppresses the rim roll-up motion which in turn suppresses geyser formation by interfering with vertical elongation, ultimately producing a highly damped drop migration and ejection along the wedge center-line. In contrast, the minimally damped drop of $\beta<\beta_{\perp}$ rolls-up along the wedge axis, forming a geyser and resulting in high amplitude 


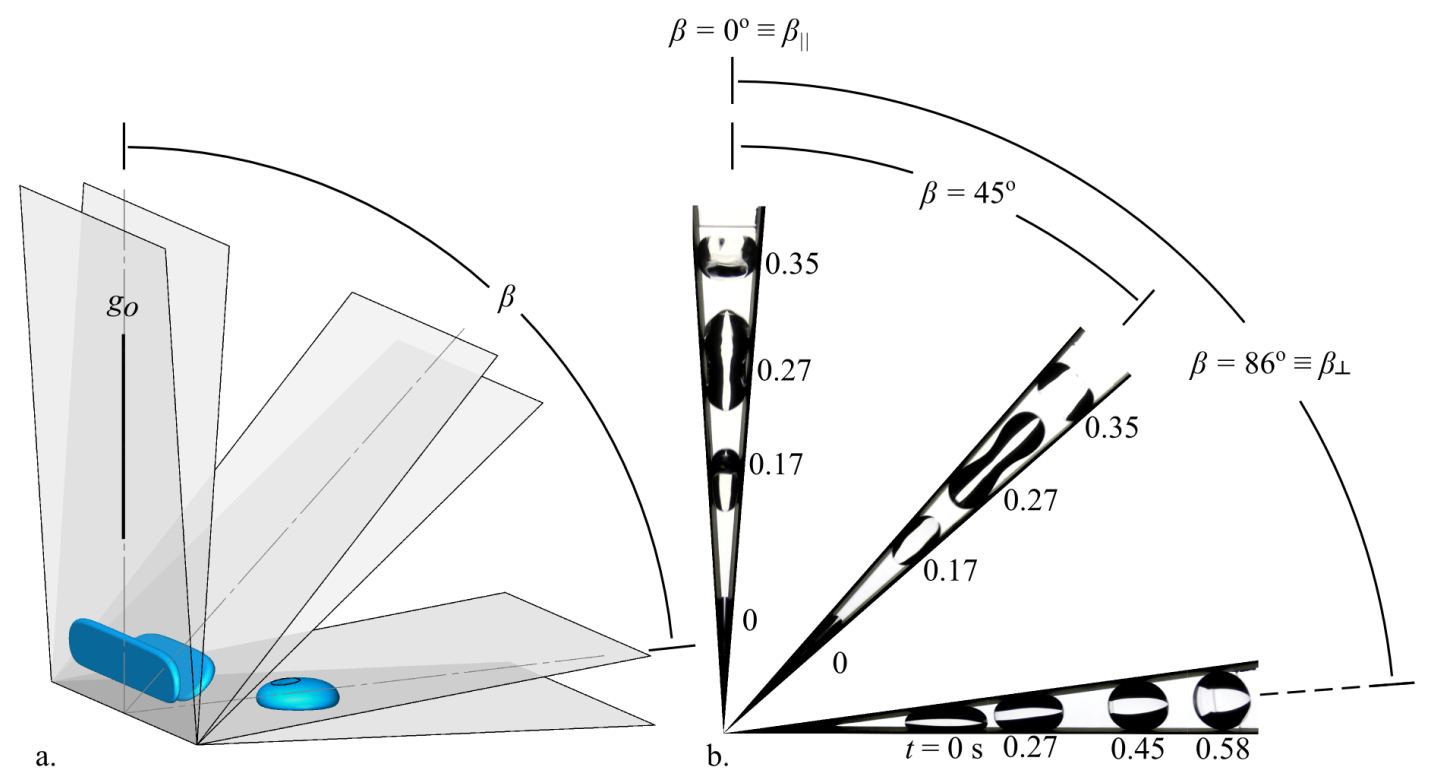

Figure 9: Effect of wedge angle $\beta$ relative to gravity on oscillation amplitude and frequency at ejection location: a. numerically computed initial shapes of $1 \mathrm{~mL}$ drops in hydrophobic wedges of $\theta=150^{\circ}$ for $\alpha=4^{\circ}$ and $\beta=0^{\circ}, 45^{\circ}$, and $86^{\circ}$, b. drop tower experiments of cases in a.

deformations and under-damped oscillations during migration and ejection, characteristics similar to puddle jumping. Numerically computed initial equilibrium conditions for three configurations of $\beta$ for a $1 \mathrm{~mL}$ drop in a wedge with $\alpha=4^{\circ}$ and $\theta=150^{\circ}$ are shown in Fig. 9a (Chen et al. 2011). Corresponding drop tower experiments are provided in Fig. 9b. The differences in drop distortion are best observed at $t=0.27 \mathrm{~s}$ with the horizontal case $\beta=86^{\circ}$ producing the least distortion.

The damping mechanism of $\beta=\beta_{\perp}$ wedges is primarily attributed to the upper confining wall which at small $\alpha$ limits vertical drop motion associated with the roll-up sequence and increases viscous dissipation of higher harmonic capillary waves during early stages of the flow. Maximum interference and dissipation for a drop in a non-wetting wedge occurs as $\alpha \rightarrow 0^{\circ}$ with $H_{w} \lesssim H$, where $H_{w}$ is the distance between the two surfaces. As demonstrated 
in the drop tower tests of Fig. 10, a $2 \mathrm{~mL}$ puddle rebounds between parallel hydrophobic substrates at various separation distances $H \lesssim H_{w} \lesssim D$, where $D$ is the drop spherical diameter. By digitally tracking the planar drop area, the characteristic time constant is $\tau=3.91 / \zeta \omega_{n}$, where $\zeta$ is the damping ratio and $\omega_{n}$ is the natural frequency for each height $H_{w}$, and is calculated from the logarithmic decrement of successive peaks from the resultant exponentially decaying sinusoidal response. The time constant $\tau$ decreases with $H_{w} / D$ as listed in Fig. 10 and as predicted under the viscous time scale $\tau_{\nu} \sim H_{w}^{2} / 4 \nu$. Experiments are primarily conducted at center-line angles $\beta=\beta_{\perp}$ since this initial condition produces the most uniformly damped ejected drops at $x_{r_{i n s}}$. The receding edge initial location for drops in such wedges is bounded by $x_{c a p} \lesssim x_{r_{o}} \lesssim x_{r_{i n s}}$, where $x_{c a p}=H / \cos \alpha \tan 2 \alpha$ approximates the receding edge location where the drop just makes contact with the upper surface. Normalizing each term in this boundary inequality by $x_{r_{i n s}}$ and subtracting each from unity produces

$$
x_{o}^{*}=1-x_{o}\left(\frac{4 \pi}{3 V}\right)^{1 / 3} \frac{\sin \alpha}{1-\sin \alpha},
$$

and

$$
x_{\text {max }}^{*} \approx 1-1.61 \mathrm{Bo}_{V}^{-1 / 2}=1-3.22 \mathrm{Bo}_{V}^{-1 / 2} \frac{\tan \alpha}{\tan 2 \alpha(1-\sin \alpha)},
$$

such that $0 \lesssim x_{o}^{*} \lesssim x_{\text {max }}^{*}$. Equation 4 provides a measure of confinement for drops satisfying $\pi H^{3} / V<1$. For drop tower experiments, maximum confinement occurs as $x_{o} \rightarrow x_{c a p}$ resulting in $x_{o}^{*} \rightarrow x_{\max }^{*}$. In the limits of small wedge half angles $\alpha \rightarrow 0$ and large drop volumes $V \rightarrow \infty$ total confinement is given as $x_{o}^{*} \rightarrow x_{\max }^{*} \rightarrow 1$. In contrast, as $x_{o}^{*} \rightarrow 0$ no confinement is achieved. Equation 4 combines variables $V, \alpha$, and $x_{o}$ into a single parameter that is employed to characterize the dependent variables of ejection time and velocity. 


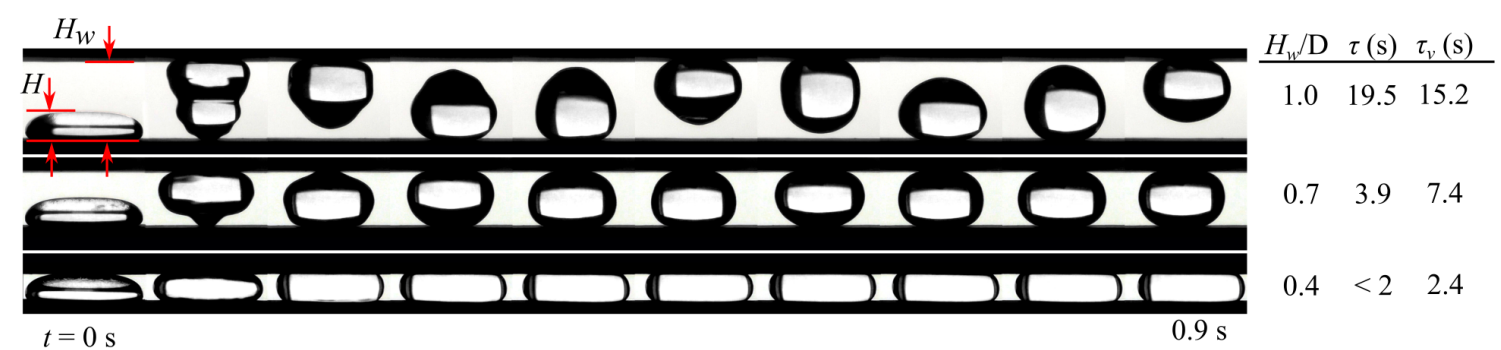

Figure 10: $10 \mathrm{~Hz}$ images from drop tower tests of $2 \mathrm{~mL}$ drops between parallel hydrophobic surfaces with varying separation height $H_{w}$. The computed time constant $\tau=3.91 / \zeta \omega_{n}$ for planar area oscillation decay decreases with the separation height $H_{w}$ as predicted by the viscous time scale $t_{\nu} \sim H_{w}^{2} / 4 \nu$

\subsection{Results and Discussion}

The transient average drop locations $x_{\text {ave }}$ for all of the experiments listed in Table A.2 are presented in Fig. 11. The wedge ejection velocities $U_{w}$ are calculated from centraldifferences and identified by linear red slopes. The red ticks mark the time $t_{w}$ at which the drops pass through the inscribed location $x_{i n s}$. Drop trajectory histories that do not include such tick marks do not fully eject within the drop time available $t_{w}>t_{d r o p}$. In all tests presented, the drop behavior is characterized by a transition to constant velocity, even for drops that do not fully achieve $x_{r_{i n s}}$. Experiments conducted that do not exhibit a linear region are not reported.

The experimental average exit velocities are normalized by the theoretical maximum velocity $\tilde{U}$ from Eq. 2 as $U_{w} / \tilde{U} \equiv U_{w}^{*}$ and plotted against the capillary confinement parameter $x_{o}^{*}$ in Fig. 12 using solid symbols for non-ejected drops and open symbols for ejected drops. Horizontal and vertical error bars provide measurement uncertainty, with an average relative uncertainty of $\pm 10 \%$. Repeatability of a $2 \mathrm{~mL}$ drop in a wedge of angle $\alpha=2.5^{\circ}$ 

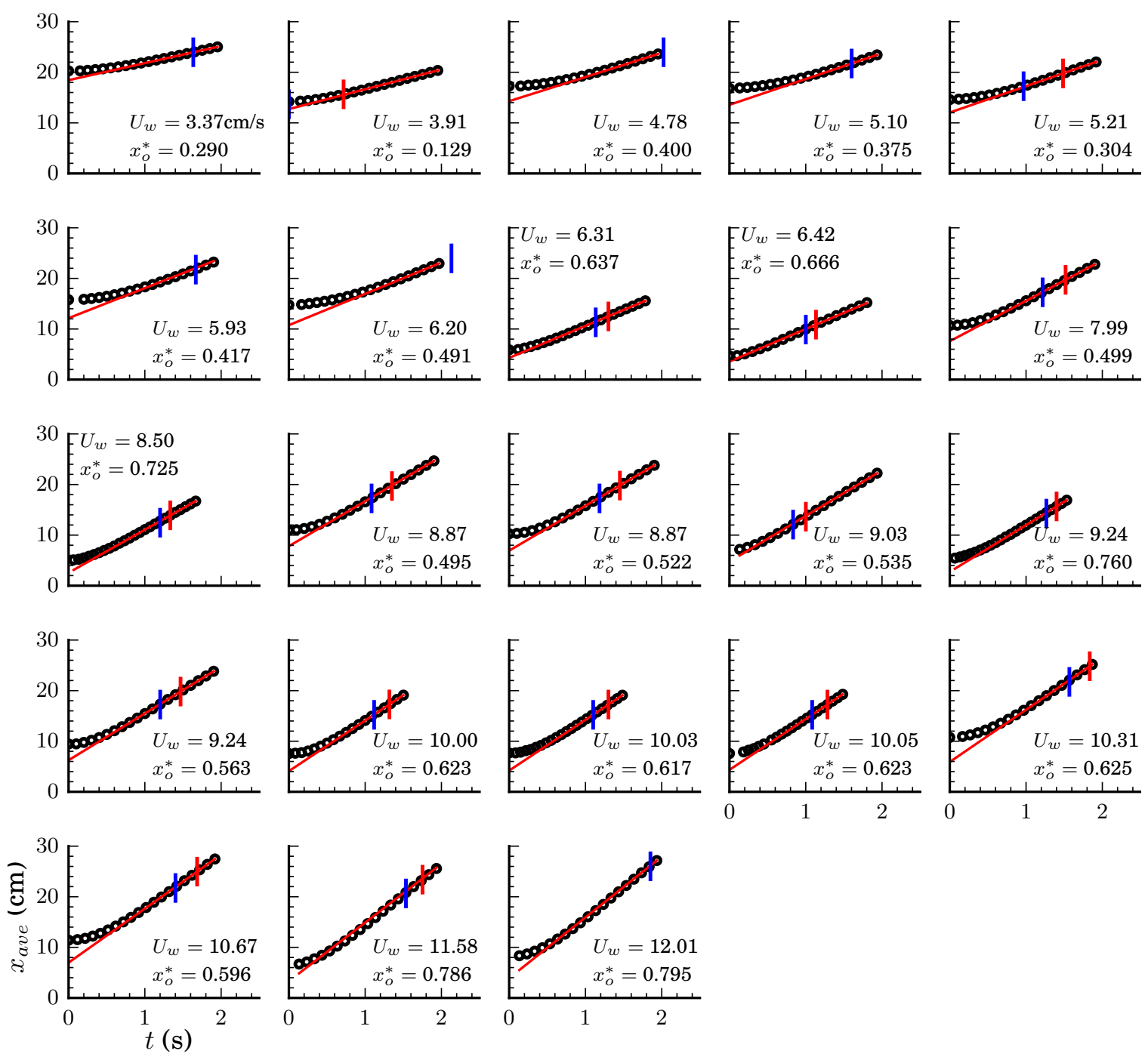

Figure 11: Transient location of averaged drop position $x_{\text {ave }}(\mathrm{cm})$ vs $t(\mathrm{~s})$ for wedges $1.0^{\circ} \leq \alpha \leq 3.8^{\circ}$ and drops $0.5 \leq V \leq 10 \mathrm{~mL}$ with varying initial locations resulting in confinements $0.1 \leq x_{o}^{*} \leq 0.80$. Plots are arranged in order of increasing maximum drop velocity $U_{w}$ which generally increases with $x_{o}^{*}$. Inscribed drop locations are noted by red tick marks when achieved. Static equilibrium locations are noted by blue tick marks. 
at $x_{o} \approx x_{c a p}$ for $x_{o}^{*}=0.62$ is observed to be $U_{w}=10.03 \pm 0.02 \mathrm{~cm} / \mathrm{s}$ for 3 tests conducted.

The predicted velocity from Eq. 2 is presented in Fig. 12 as small, closed dot-symbols. The model over-predicts velocity for all $x_{o}^{*}$ as expected, but with decreasing error as $x_{o}^{*} \rightarrow 1$ where the model assumptions are most appropriate. An average model discrepancy of $\pm 67 \%$ is achieved without consideration of complex effects due to transients, refined geometry, moving contact line dynamics, and others. An approximate linear trend is observed for both

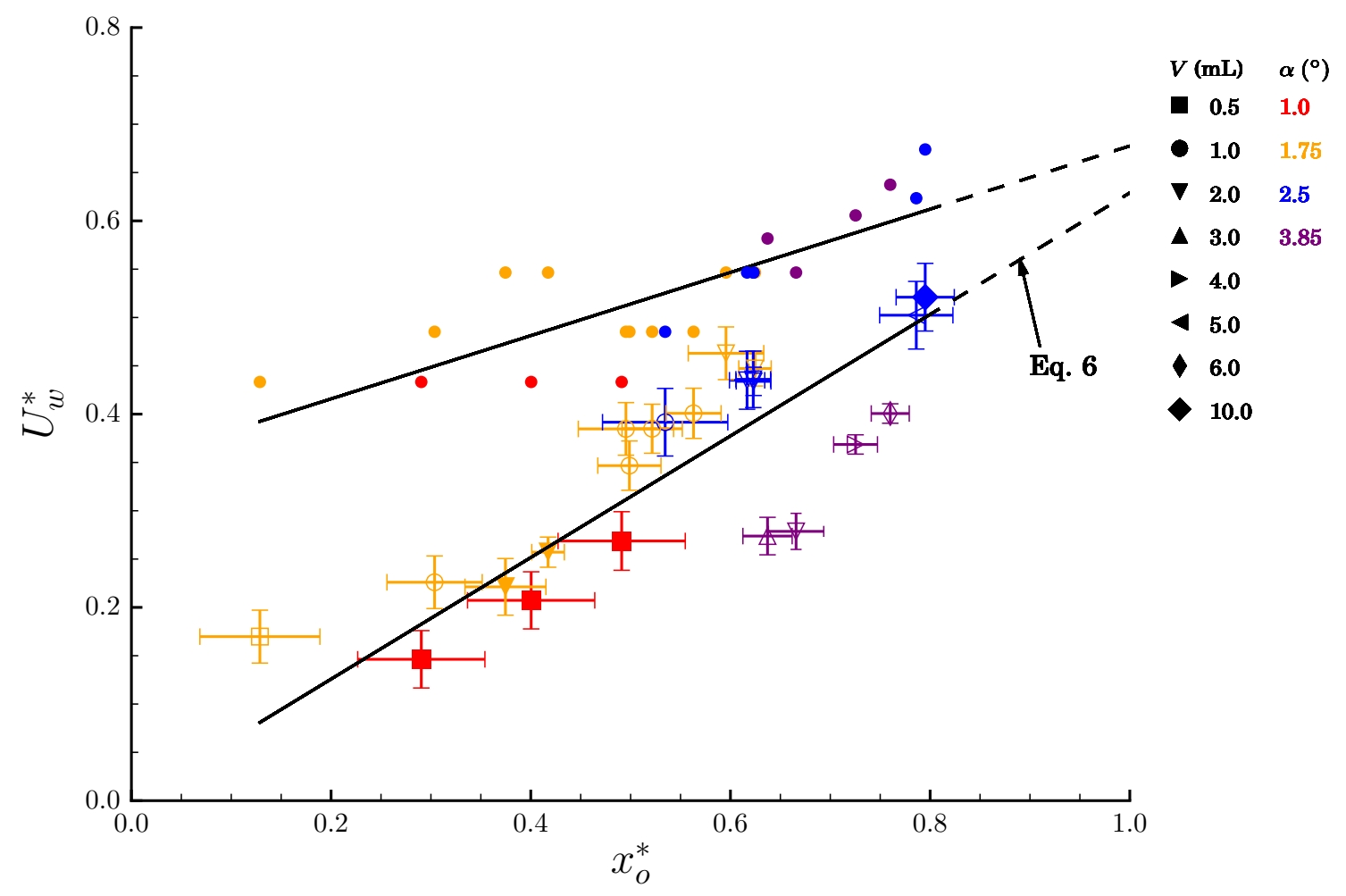

Figure 12: Normalized drop velocities $U_{w}^{*}=U_{w} / \tilde{U}$ versus capillary confinement parameter $x_{o}^{*}$. Open and solid symbols imply drop ejection or no-ejection, respectively. Small, closed dot-symbols without error bars identify theoretical velocities from the cylindrical puddle surface energy difference approximation of Eq. 2. An approximate linear trend in both experimental and theoretical velocities tend towards agreement as $x_{o}^{*} \rightarrow 1$. 
experimental and theoretical velocities in Fig. 12, the experimental data being correlated for wedge design purposes by

$$
U_{w_{l}}^{*}=0.64 x_{o}^{*}
$$

providing a quick estimate with $\pm 18 \%$ average error.

Ejection times for drops that achieve $x_{r_{i n s}}$ within $t_{d r o p}$ are presented in Fig. 13. Predicted ejection times $t_{w}$ from Eq. 3 are plotted with the data in Figure 13a. As anticipated, the Eq. 3 ejection times are shorter than observed in the experiments. A correction to Eq. 3 of the form

$$
t_{w_{\text {corr }}}=1.82\left(\frac{\rho R_{s}^{4}}{4 \sigma g_{o}}\right)^{1 / 4}(\csc \alpha-1)
$$

yields an average error of $\pm 14 \%$ as shown in Fig. 13b. Additionally, using the linear model ejection velocities $U_{w_{l}}=0.64 \tilde{U} x_{o}^{*}$ from Eq. 6 and the distances to the inscribed locations $x_{r_{i n s}}-x_{r_{o}} \equiv \Delta x$, the form of Eq. 7 is re-derived from $t_{w_{l}}=\Delta x / U_{w_{l}}$ yielding

$$
t_{w_{l}}=1.56\left(\frac{\rho R_{s}^{4}}{4 \sigma g_{o}}\right)^{1 / 4}(\csc \alpha-1)
$$

with an average error of $\pm 16 \%$, as shown in Fig. 13c. No specific trend in ejection times are found with respect to $x_{o}^{*}$. However the form of Eq. 8 derived from the linear capillary confinement parameter ejection velocity model closely matches the corrected ejection time of Eq. 7 which is derived from first principles. Thus, either Eq. 7 or 8 will serve adequately for wedge drop generator design.

\subsection{Conclusions}

As introduced in this report, the hydrophobic wedge geometry provides an attractive degree of control for use as a passive, large volume, drop generator. The nearly non-oscillating 


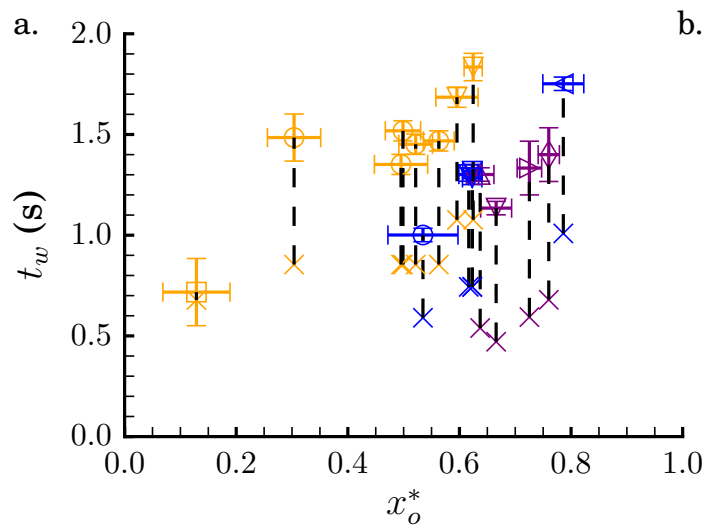

b.

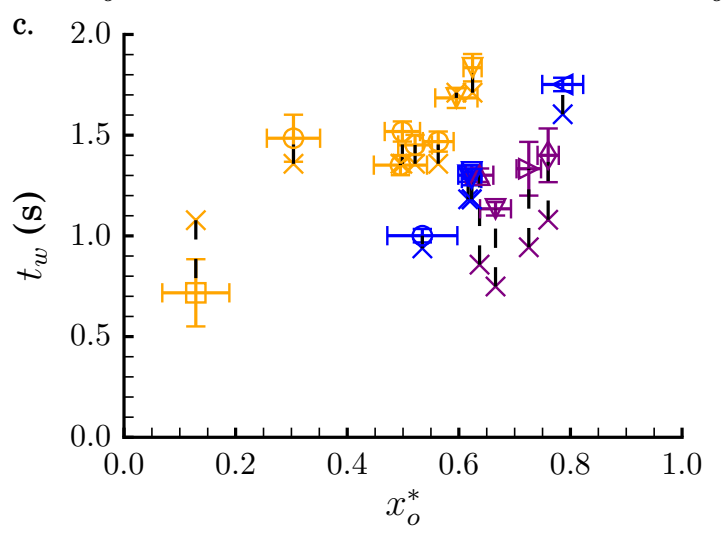

Figure 13: Experimental and theoretical ejection times $t_{w}$ with respect to the capillary confinement parameter $x_{o}^{*}$ with a. Eq. 3, b. Eq. 7, and c. Eq. 8.

drops generated during the drop tower study range in volumes from $0.5 \leq V \leq 10 \mathrm{~mL}$ with ejection velocities between $3.37 \leq U_{w} \leq 12.01 \mathrm{~cm} / \mathrm{s}$ and oscillation amplitudes typically less than $3 \%$ of spherical radius dimension at ejection. Without the time limitation of a drop tower, significantly larger and slower drops could be ejected for a variety of fundamental and applied investigations. For example, low speed impacts of large non-oscillating drops on heated surfaces above the Leidenfrost temperature could be uniquely studied employing the hydrophobic wedge geometry.

The results provided suggest the use of the capillary confinement parameter $x_{o}^{*}$ and its correlations to predict wedge drop generator performance. The confinement parameter com- 
bines drop volume, drop initial condition/position, and wedge half-angle into a single term that can be used to approximate drop ejection velocity and ejection time for a hydrophobic wedge (Eqs. 6 and 8, respectively). Alternatively, the semi-empirical ejection time of Eq. 7 also provides predictions based on an ideal energy transfer velocity limit, closely matching the empirically derived ejection model time Eq. 8. For the 23 nominal drop tests presented, average prediction uncertainties in Eqs. 6-8 are less than $\pm 20 \%$. With such tools in hand, one can expect to eject drops of volume $V \lesssim 2 \mathrm{~mL}$ with ejection velocities $U_{w} \lesssim 9 \mathrm{~cm} / \mathrm{s}$ in $0.5 \mathrm{~s}$ drop towers, $V \lesssim 10 \mathrm{~mL}$ at $U_{w} \lesssim 12 \mathrm{~cm} / \mathrm{s}$ in $2 \mathrm{~s}$ towers, and $V \lesssim 30 \mathrm{~mL}$ at $U_{w} \lesssim 13$ $\mathrm{cm} / \mathrm{s}$ in $5 \mathrm{~s}$ towers. 


\section{Applications and Future Work}

\subsection{Future planar wedge drop migration investigations}

The work presented here serves as a foundation for continued investigations in drop migration in non-wetting tapered geometries. The energy model presented is a first order/first principle approximation, accounting for inertial and capillary energies over viscous losses and contact line hysteresis. The drop time in all cases presented is less than the viscous time scale $t_{d r o p}<t_{\nu}$. Future work may seek to expand the analytic model to such effects to increase accuracy as well as adapt the model for fluids of various viscosities. Additionally, identification of the no-ejection limit for wedge bound drops is of interest, and expected as drop volume $V$ and wedge half-angle $\alpha$ decrease. A few experiments regarding these topics were carried out as inspiration for future work. Figure 14b presents snapshots of five drop tests of $1 \mathrm{~mL}$ drops in an $\alpha=4^{\circ}$ wedge with varying viscosity $\nu$. As expected, increased viscosity serves to reduce ejection velocity for drops of the same initial confinement parameter $x_{o}^{*}$. The Fig. 14a time sequence of a small volume $V=0.2 \mathrm{~mL}$ drop inside a $\alpha=2.7^{\circ}$ wedge

demonstrates a no-ejection regime where the drop transitions to a final resting location $x_{r_{f}}$ set by contact angle hysteresis, in contrast to the inertial-capillary ejected drops studied throughout the preceding sections.

Other tapered hydrophobic geometries, such as a cone or rectangular diffuser, are also of interest for future investigation. A tapered hydrophobic cone varies from the wedge in a few interesting ways. For instance, a capillary drop will occlude the entire cross section of a cone, restricting air flow from the advancing side to the receding side of the drop. In order for migration to occur, air must flow through the vertex of the cone or around the 
drop along the hydrophobic surface. The drop migration velocity is thus air flow limited. This feature can be used to reduce the drop migration velocity to nearly zero. A single demonstrate of a conical drop generator is shown in Fig. 15. A 3D printed cone with $\alpha=2^{\circ}$ in Fig. $15 \mathrm{~b}$ is shown in the Fig. $15 \mathrm{c}$ snapshot with a $0.71 \mathrm{~mL}$ drop ejecting with steady velocity $7.2 \mathrm{~cm} / \mathrm{s}$.
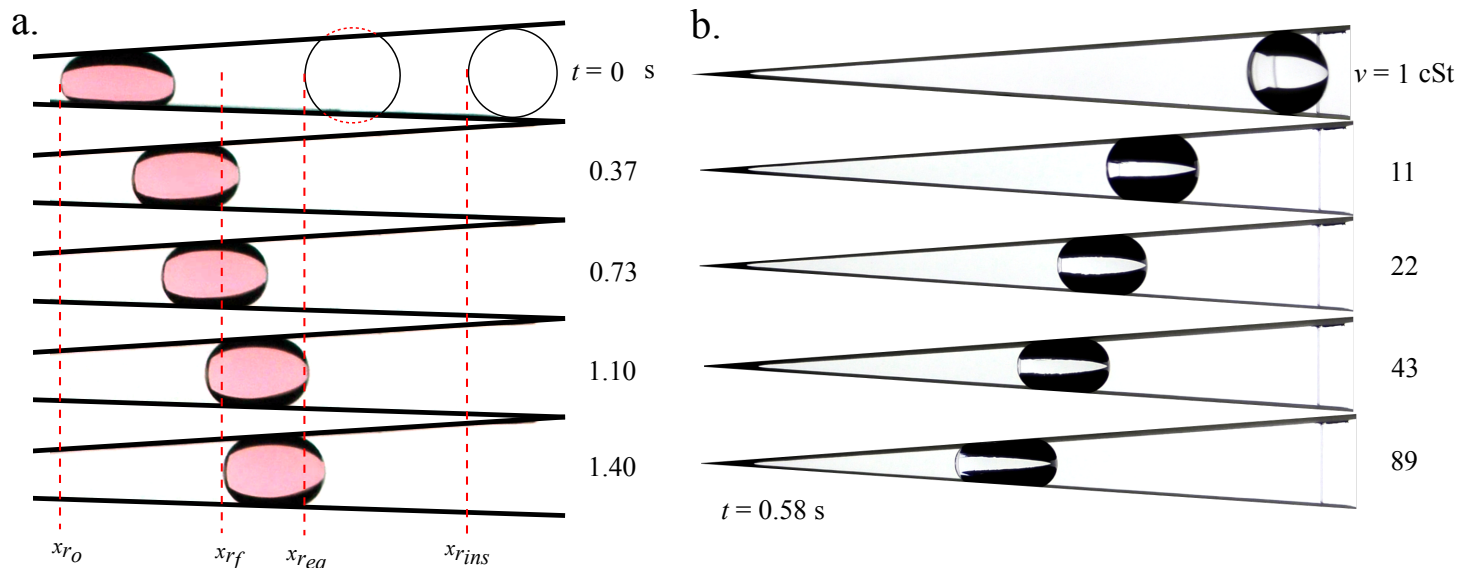

Figure 14: a. Time sequence of a $0.2 \mathrm{~mL}$ drop in hydrophobic wedge of $\alpha=2.8^{\circ}$ during a drop tower experiment. By $t=1.4 \mathrm{~s}$ the drop has been slowed and stopped by dissipative forces. $\mathrm{b}$. Snapshot at $t=0.58 \mathrm{~s}$ of $1 \mathrm{~mL}$ viscous drops ranging in kinematic viscosity from 1 to $89 \mathrm{cSt}$. Increased viscosity decreases the viscous time scale $t_{\nu}=L_{s}^{2} / \nu$, thus initiating early transition to constant velocity as well as decreased ejection velocity.

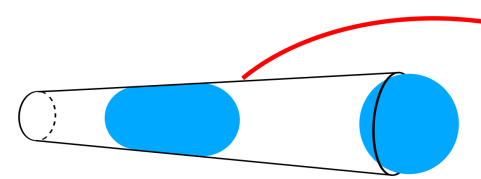

a.

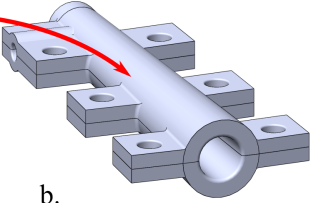

b.

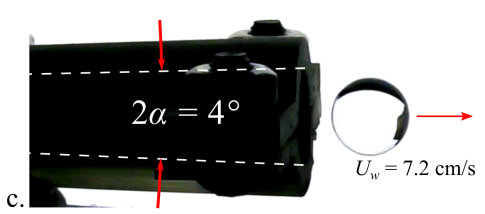

Figure 15: a. A conical drop generator is designed and 3D printed in b. as two halves such that the internal surface of each can be coated with a hydrophobic treatment. c. A single snap shot of a $0.71 \mathrm{~mL}$ drop ejecting from the $\alpha=2^{\circ}$ cone with velocity $U_{w}=7.2 \mathrm{~cm} / \mathrm{s}$. 


\subsection{Wedge drop generating devices}

The long term goal for this work is to implement the wedge drop generator into capillary drop studies where large quiescent slow moving drops are required, particularly for space experiments where the duration of micro-gravity is essentially unlimited. Such an environment is conducive to large sample size experiments, which require continuous deployment of identical drops. Integration of a hydrophobic wedge and a fluid injection system could meet such a requirement. Figure 16a presents a simple schematic for a system using a hy-

drophobic wedge and syringe pump. Fluid is injected at a steady rate $Q_{\text {in }}$ near the apex of the wedge where it grows into a large drop until detachment occurs and the drop migrates away under the capillary pressure of Eq. 1. Figure 16b provides a drop tower demonstration of Fig. 16a. A single snapshot captures 3 drops of volume $V=0.29 \pm 0.01 \mathrm{~mL}$ in an $\alpha=4^{\circ}$ wedge at stages of (1) growth, (2) detachment, and (3) ejection, with eventual ejection velocities of $U_{w}=10.2 \pm 0.1 \mathrm{~cm} / \mathrm{s}$ for the drops from a constant water flow rate of $Q_{\text {in }}=48 \mathrm{~mL} / \mathrm{min}$. Further experiments could investigate the effect of wedge geometry, fluid properties, and injection rate on characteristics such as ejection velocity, ejection time, and drop oscillation, frequency, and amplitude.

Aside from continuous drop deployment, a drop-on-demand device could integrate the wedge geometry into a hand-held "drop shooter" for astronaut experiments. The Fig. 17a-c schematics outline a 3 step hand controlled process. In Fig. 17a a drop of desired volume is deposited between two parallel planar hydrophobic surfaces, with a prescribed initial separation $H$. In Fig. 17b, the top surface is rotated using an adjustment mechanism until the downstream opening reaches the required diameter for the injected drop $2 R_{s}$. Finally, in Fig. 17c, the drop passively migration from the confined end due to the internal capillary 


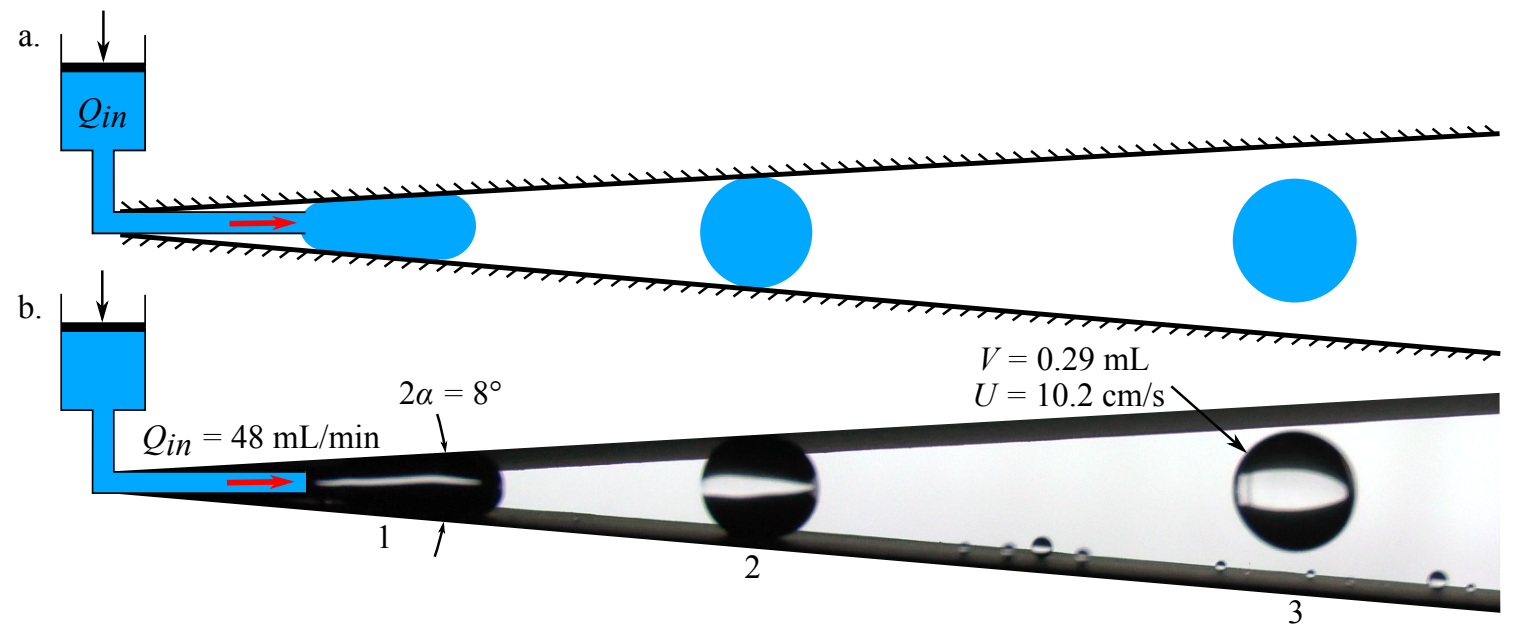

Figure 16: a. Example schematic of a continuous wedge drop generator through growth and detachment of drops at the apex of a planar hydrophobic wedge. b. Snapshot of drop tower experiment where a stream of $Q_{i n}=48 \mathrm{~mL} / \mathrm{min}$ of water is injected into an $\alpha=4^{\circ}$ wedge and rectified into drops of volume $V=0.29 \mathrm{~mL}$ with ejection velocity $U_{w}=10.2 \mathrm{~cm} / \mathrm{s}$.

pressure gradient, and subsequently ejects at the opening. A device of this kind provides unique user input as well as a wide range of ejection volumes and velocities to accommodate the desired investigation or demonstration.

\subsection{Concluding remarks}

The wedge drop generator provides an attractive simple method for large quiescent drop generation not readily achieved by other drop generation techniques. It is desired that the work conducted in this thesis is carried on and applied beyond the efforts of the author, and that the avenues discussed in this section provide inspiration for future drop tower research. 


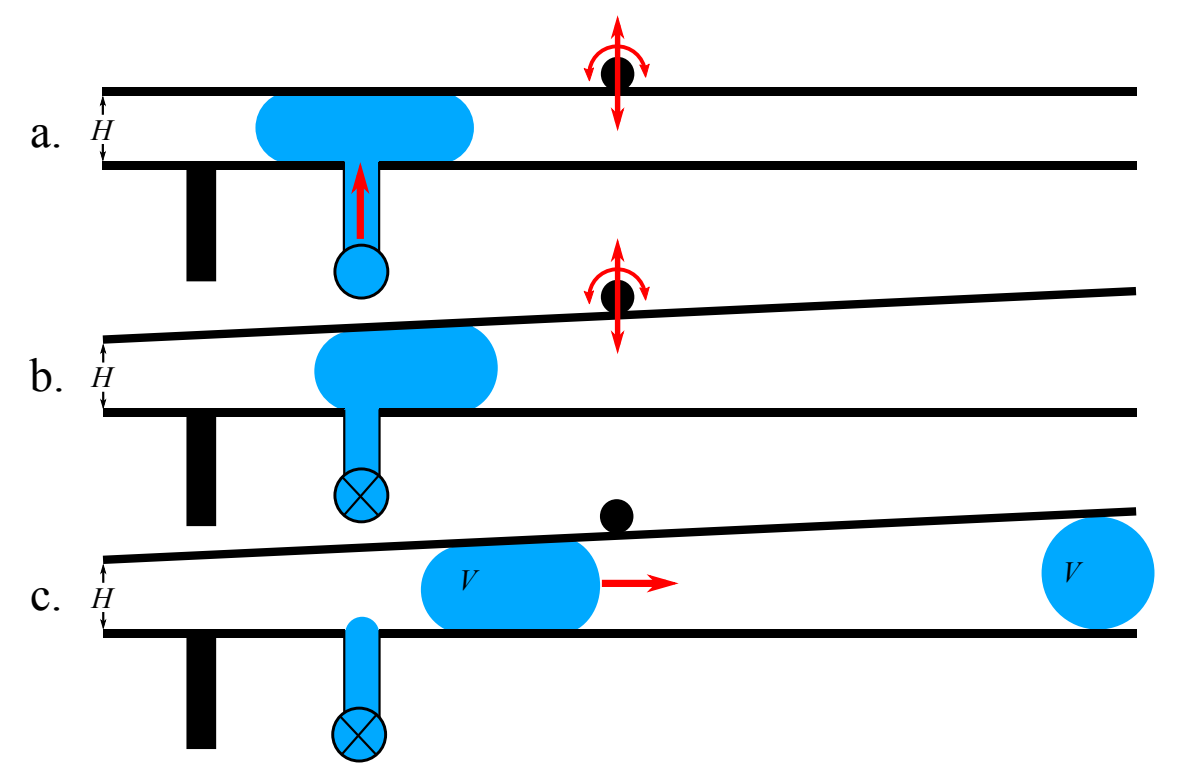

Figure 17: Simple schematic for a hand-held wedge drop generator. a. Fluid is injected between parallel hydrophobic surfaces until the desired volume is deposited. b. The upper surface is then mechanically rotated to the desired angle until in c. the deposited drop migrates and ejects under the internal capillary pressure from Eq. 1. 


\section{References}

Al Jubaree T, Weislogel M, Hua T (2016) Viscous Puddle Jump. In 69th Annual Meeting of the APS Division of Fluid Dynamics, p 108

Attari B, Weislogel M, Wollman A, Chen Y, Snyder T (2016) Puddle Jumping : Spontaneous Ejection of Large Liquid Droplets from Hydrophobic Surfaces During Drop Tower Tests. Physics of Fluids 28: 102104

Avhad A, Jubaree T, Torres L, Weislogel M, Tan H (2017) Numerical analysis of puddle jumping from hydrophobic surface. In 70th Annual Meeting of the APS Division of Fluid Dynamics 326

Baratian D, Cavalli A, Van Den Ende D, Mugele F (2015) On the shape of a droplet in a wedge: new insight from electrowetting. Soft Matter 11: 7712-77721

Chen Y, Schaffer B, Weislogel M, Zimmerli G (2011) Introducing se-fit: Surface evolver-fluid interface tool for studying capillary surfaces. In 49th AIAA Aerospace Sciences Meeting including the New Horizons Forum and Aerospace Exposition 1319

Concus P, Finn R (1998) Discontinuous behavior of liquids between parallel and tilted plates. Physics of Fluids 10: 39-43

Dangla R, Kayi S, Baroud C (2013) Droplet microfluidics driven by gradients of confinement. Proceedings of the National Academy of Sciences 110: 853-858

Jenson R, Wollman A, Weislogel M, Sharp L, Green R, Canfield P, Klatte J, Dreyer M 
(2014) Passive phase separation of microgravity bubbly flows using conduit geometry. International Journal of Multiphase Flow 65: 68-61

Khare K, Herminghaus S, Baret J, Law B, Brinkmann M, Seemann R (2007) Switching liquid morphologies on linear grooves. Langmuir 23: 12997-13006

Langbein DW (2002) Capillary surfaces: shape - stability - dynamics, in particular under weightlessness volume 178 Springer Science \& Business Media

Luo C, Heng X (2014) Separation of oil from a water/oil mixed drop using two nonparallel plates. Langmuir 30: 10002-10010

Luo C, Heng X, Xiang M (2014) Behavior of a liquid drop between two nonparallel plates. Langmuir 30: 8373-8380

Popinet S (2003) Gerris: a tree-based adaptive solver for the incompressible euler equations in complex geometries. Journal of Computational Physics 190: 572-600

Reyssat E (2014) Drops and bubbles in wedges. Journal of Fluid Mechanics 748: 641-662

Richard D, Quéré D (2000) Bouncing water drops. EPL (Europhysics Letters) 50: 769

Robinson DW, Chai A (1997) Development of a device to deploy fluid droplets in microgravity. NASA Technical Reports Server

Ruiz-Gutiérrez E, Semprebon C, McHalen G, Ledesma-Aguilar R (2017) Statics and Dynamics of Liquid Barrels in Wedge Geometries. Journal of Fluid Mechanics 842

Schindelin J, Arganda-Carreras I, Frise E, Kaynig V, Longair M, Pietzsch T, Preibisch S, Rueden C, Saalfeld S, Schmid B, Tinevez JY, White DJ, Hartenstein V, Eliceiri K, 
Tomancak P, Cardona A (2012) Fiji: an open-source platform for biological-image analysis. Nature Methods 9: 676-682

Schmidt E, Weislogel M (2016) Spontaneous Droplet Jump with Electro-Bouncing. In 69th Annual Meeting of the APS Division of Fluid Dynamics, p 393

Weislogel M, Jenson R, Chen Y, Collicott S, Klatte J, Dreyer M (2009) The capillary flow experiments abourd the International Space Station: Status. Acta Astronautica 65: $861-869$

Weislogel M, Wollman A, Jenson R, Geile J, Tucker J, Wiles B, Trattner A, Devoe C, Sharp L (2015) Capillary Channel Flow ( CCF ) EU2 02 on the International Space Station ( ISS ): An Experimental Investigation of Passive Bubble Separations in an Open Capillary Channel. NASA NTRS Technical Reports

Wollman A, Weislogel M, Wiles B, Pettit D, Snyder T (2016) More investigations in capillary fluidics using a drop tower. Experiments in Fluids 57: 1-17

Xu W, Lan Z, Peng B, Wen R, Chen Y, Ma X (2016) Directional Movement of Droplets in Grooves: Suspended or Immersed?. Scientific reports 6: 18836 


\section{Appendix}

\section{Appendix A}

Table 2: Range of variables, parameters, and responses from nominal wedge experiments.

\begin{tabular}{|c|c|c|c|c|c|c|c|}
\hline & $\begin{array}{c}2 \alpha \\
\left({ }^{\circ}\right) \pm 5 \%\end{array}$ & $\begin{array}{c}\beta \\
\left({ }^{\circ}\right) \pm 5 \%\end{array}$ & $\begin{array}{c}V \\
(m L) \pm 2 \%\end{array}$ & $\begin{array}{c}x_{r o} \\
(\mathrm{~cm}) \pm 5 \%\end{array}$ & $\begin{array}{c}x_{o}^{*} \\
\pm 10 \%\end{array}$ & $\begin{array}{c}U_{w} \\
(\mathrm{~cm} / \mathrm{s}) \pm 10 \%\end{array}$ & $\begin{array}{c}t_{w} \\
\text { (s) } \pm 10 \%\end{array}$ \\
\hline No. 1 & 2.0 & 89.0 & 0.50 & 14.75 & 0.491 & 6.20 & NA \\
\hline 2 & 2.0 & 89.0 & 0.50 & 17.28 & 0.400 & 4.78 & NA \\
\hline 3 & 2.0 & 89.0 & 0.50 & 20.28 & 0.290 & 3.37 & NA \\
\hline 4 & 3.5 & 88.3 & 0.50 & 14.20 & 0.129 & 3.91 & 0.88 \\
\hline 5 & 3.5 & 88.3 & 1.00 & 9.42 & 0.522 & 8.87 & 1.52 \\
\hline 6 & 3.5 & 88.3 & 1.00 & 9.45 & 0.563 & 9.24 & 1.53 \\
\hline 7 & 3.5 & 88.3 & 1.00 & 10.69 & 0.499 & 7.99 & 1.60 \\
\hline 8 & 3.5 & 88.3 & 1.00 & 10.96 & 0.495 & 8.87 & 1.43 \\
\hline 9 & 3.5 & 88.3 & 1.00 & 14.59 & 0.304 & 5.21 & 1.60 \\
\hline 10 & 3.5 & 88.3 & 2.00 & 10.75 & 0.625 & 10.31 & 1.94 \\
\hline 11 & 3.5 & 88.3 & 2.00 & 11.42 & 0.596 & 10.67 & 1.75 \\
\hline 12 & 3.5 & 88.3 & 2.00 & 15.78 & 0.417 & 5.93 & NA \\
\hline 13 & 3.5 & 88.3 & 2.00 & 16.84 & 0.375 & 5.10 & NA \\
\hline 14 & 5.0 & 87.5 & 1.00 & 7.17 & 0.535 & 9.03 & 1.07 \\
\hline 15 & 5.0 & 87.5 & 2.00 & 7.56 & 0.623 & 10.00 & 1.38 \\
\hline 16 & 5.0 & 87.5 & 2.00 & 7.57 & 0.623 & 10.05 & 1.35 \\
\hline 17 & 5.0 & 87.5 & 2.00 & 7.68 & 0.617 & 10.03 & 1.37 \\
\hline 18 & 5.0 & 87.5 & 5.00 & 6.70 & 0.786 & 11.58 & 1.84 \\
\hline 19 & 5.0 & 87.5 & 10.00 & 8.35 & 0.795 & 12.01 & NA \\
\hline 20 & 7.7 & 86.2 & 2.00 & 4.68 & 0.666 & 6.42 & 1.25 \\
\hline 21 & 7.7 & 86.2 & 3.00 & 5.89 & 0.637 & 6.31 & 1.17 \\
\hline 22 & 7.7 & 86.2 & 4.00 & 5.05 & 0.725 & 8.50 & 1.47 \\
\hline 23 & 7.7 & 86.2 & 6.00 & 5.56 & 0.760 & 9.24 & 1.53 \\
\hline
\end{tabular}

Table 3: Range of variables used for a. parallel wedge and b. initial condition experiments.

\begin{tabular}{cccccc|cccc} 
a. & $\begin{array}{c}\mathbf{2 \alpha} \\
\left({ }^{\circ} \pm \mathbf{5 \%}\right.\end{array}$ & $\begin{array}{c}\boldsymbol{V} \\
(\mathbf{m L}) \pm \mathbf{2 \%}\end{array}$ & $\begin{array}{c}\boldsymbol{H}_{\boldsymbol{w}} \\
(\mathbf{c m}) \pm \mathbf{5 \%}\end{array}$ & $\begin{array}{c}\boldsymbol{H}_{\boldsymbol{w}} / \boldsymbol{D}_{\boldsymbol{s}} \\
\pm \mathbf{1 0} \%\end{array}$ & $\begin{array}{c}\boldsymbol{\tau} \\
(\mathbf{s}) \pm \mathbf{2 5 \%}\end{array}$ & $\mathbf{b .}$ & $\begin{array}{c}\mathbf{2} \boldsymbol{\beta} \\
\left({ }^{\circ}\right) \pm \mathbf{5 \%}\end{array}$ & $\begin{array}{c}\boldsymbol{\beta} \\
\left({ }^{\circ}\right) \pm \mathbf{5 \%}\end{array}$ & $\begin{array}{c}\boldsymbol{V} \\
(\mathbf{m L}) \pm \mathbf{2 \%}\end{array}$ \\
\hline \hline No. 1 & 0.0 & 1.0 & 0.62 & 1.0 & 19.5 & 8.0 & 86.0 & 1.0 \\
$\mathbf{2}$ & 0.0 & 1.0 & 1.09 & 0.7 & 3.9 & 8.0 & 45.0 & 1.0 \\
$\mathbf{3}$ & 0.0 & 1.0 & 1.56 & 0.4 & $<2$ & 8.0 & 90.0 & 1.0
\end{tabular}




\section{Appendix B}

The surface energy of a confined droplet can be estimated in the limit of small $\alpha$ and large $V$ where the initial shape is simplified as a cylinder with height $H$ and radius $R_{c}$ as shown in Fig. 8a. Ignoring dissipation, the surface energy difference from confined state 1 to free state 2 is converted to kinetic motion of the bulk droplet $K E_{2}=S E_{1}-S E_{2}$ where the surface energies $S E_{i}$ are computed by $S E_{1}=\left[(\sigma A)_{l s}+(\sigma A)_{l g}\right]_{1}$ and $S E_{2}=\left[(\sigma A)_{l g}+(\sigma A)_{s g}\right]_{2}$ and the kinetic energy by $K E_{2}=\rho V U_{w}^{2} / 2$. Interface areas are calculated from the idealized geometry of Fig. 8 as discussed above where $H=2\left(\sigma / \rho g_{o}\right)^{1 / 2}, R_{c}=(V / \pi H)^{1 / 2}$, and $R_{s}=(3 V / 4 \pi)^{1 / 3}$ where $A_{l s_{1}}=2 \pi R_{c}^{2}, A_{l g_{1}}=2 \pi R_{c} H_{c a p}, A_{l g_{2}}=4 \pi R_{s}^{2}$, and $A_{s g_{2}}=2 \pi R_{c}^{2}$. Exploiting the Young-Laplace relation $\sigma_{l s}-\sigma_{s g}=-\sigma \cos \theta$ the velocity of the droplet at the inscribed point can be solved to find

$$
U_{w}=\tilde{U}\left[-\cos \theta+\left(\frac{\pi H^{3}}{V}\right)^{1 / 2}-\frac{6^{2 / 3}}{2}\left(\frac{\pi H^{3}}{V}\right)^{1 / 3}\right]^{1 / 2}
$$

where $\tilde{U}=\left(4 \sigma g_{o} / \rho\right)^{1 / 4}$ is the theoretical maximum velocity of the droplet in the limit of large puddles $\pi H^{3} / V<<1$ and high contact angles $\theta \approx 180^{\circ}$. Eq. 2 resembles that derived in Attari et al. (2016), except for the contact angle term which accounts for reduced energy due to presence of the second upper surface. An approximation for ejection time can be solved using $t_{w}=x_{i n s} / \tilde{U}$, where $x_{i n s}=R_{s}(\csc \alpha-1)$ such that

$$
t_{w}=\left(\frac{\rho R_{s}^{4}}{4 \sigma g_{o}}\right)^{1 / 4}(\csc \alpha-1)
$$

